LBL- -24914

DE89 013109

\title{
FMG, RENUM, LINEL, ELLFMG, ELLP, and DIMES: Chain of Programs for Calculating and Analyzing Fluid Flow through Two-Dimensional Fracture Networks- Theory and Design
}

Daniel Billaux, Sorin Bodea, and Jane Long

\author{
Earth Sciences Division \\ Lawrence Berkeley Laboratory \\ University of California \\ Berkeley, CA 94720
}

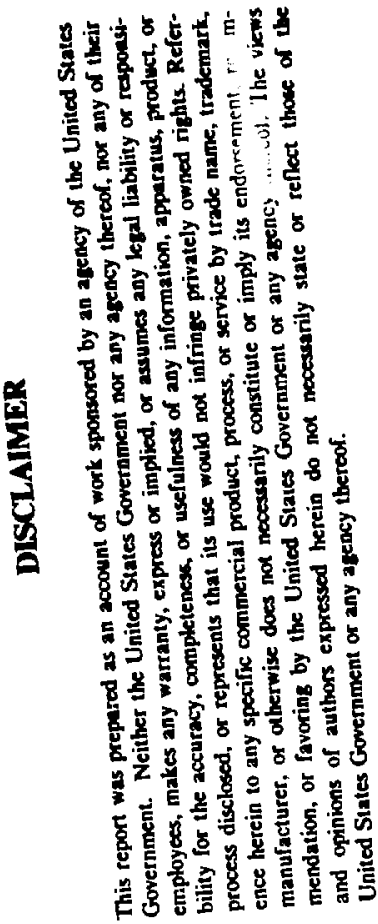

Feloruary 1988

This work was supported by the Repository and Technology Program of the Office of Civilian Radioactive Waste Management of the U.S. Department of Energy under Contract No. DE-AC03-76SF00098.

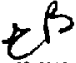




\begin{abstract}
This report describes some of the programs developed at Lawrence Berkelcy Laboratory for network modelling. By themselves, these programs form a complete chain for the study of the equivalent permeability of two-dimensional fracture networks.

FMG generates the fractures considered as line discontinulties, with any desired distribution of aperture, length, and orientation. The locations of these fractures on a planc can be either specified or generated randomly. The intersections of these fractures with each other, and with the boundaries of a specified flow region, are determined, and a finite element line network is output.

RENUM is a line network optimizer. Nodes very close to each other ane merged, deadends are removed, and the nodes are then renumbered in order to minimize the bandwidth of the corresponding linear system of equations.

LINEL computes the steady state flux througin a mesh of line elements previously processed by program RENUM. Equivalent directional permeabilities are output.

ELLFMG determines the three components of the permeability tensor which best fits the directional permeabilities output by LINEL. A measure of the goodness fit is also computed.

Two plotting programs, DIMES and ELLP, help visualize the outputs of these programs. DIMES plots the line network at various stages of the process. ELLP plots the equivalent permeability results.
\end{abstract}




\section{Table of Contents}

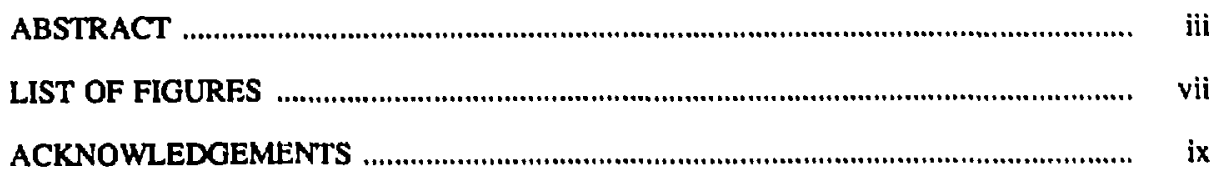

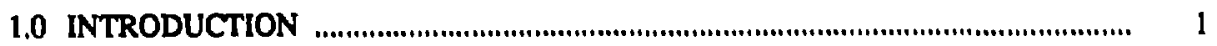

2.0 FRACTURE MESH GENERATION PROGRAM FMG _........................................... s

2.1 Generation of Fracture System ........................................................................

2.1.1 Fracture System Characteristics ............................................................. 7

2.1.2 Equations of Fncture Lines ................................................................ $\quad 12$

2.1.3 Truncation at Boundary .................................................................. $\quad 12$

2.1.4 Statistical Calculations …................................................................ 12

2.2 Fracture System in the Flow Region ...............................................................

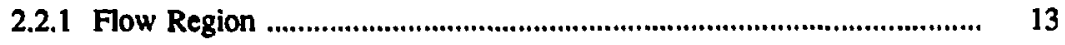

2.2.2 Fractures in Flow Region .........................................................

2.2.3 Fractures Inflow Region - Circular Case ............................................ 18

2.2.4 Statistical Calculations …................................................................ 22

2.2.5 Connections between Sides .............................................................. 22

2.3 Fracture System to be Used in Flow Model ..................................................... 23

2.3.1 Calculation of Fracture Intersections .................................................... 23

2.3.2 Elimination of Nonconducting Fractures .............................................. 24

2.3.3 Boundary Conditions and Finite Element Mesh .................................... 25

2.3.4 Boundary Conditions for Circular Networks ........................................ 28

2.3.5 Finite Element Mesh ...................................................................... $\quad 28$

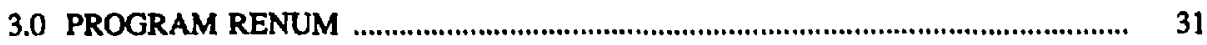

3.1 Merging Endpoints of Short Elements ............................................................ 34

3.2 Discarding Dead-ends ....................................................................................... 34

3.3 Node Renumbering and Output ........................................................................ 43

3.3.1 Banded Matrices .................................................................................. 43

3.3.2 The Cuthill-McKee Algorithm ............................................................ 43 


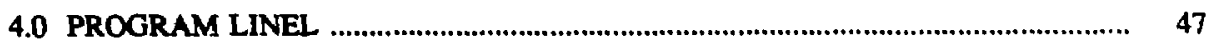

4.1 Building the Linear System of Equations ................................................... 47

4.1.1 Goveming Equations ................................................................... 47

4.1 .2 Linear System ......................................................................... 48

4.1 .3 Implementation .......................................................................... 50

4.2 Solving the Linear System ..................................................................... 52

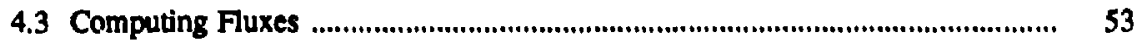

4.3.1 Flow Region .......................................................................... 53

4.3.2 Study Regions ........................................................................ 54

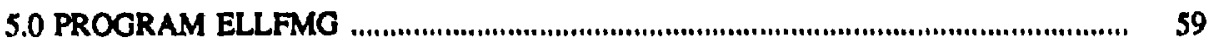

5.1 Permeability Ellipse and Permeability Distributions ....................................... 59

5.2 Finding the Permeability Parameters ................................................................ 63

5.3 Principal Permeabilities and Directions ..................................................... 67

5.4 Mean Square Error .................................................................................... 69

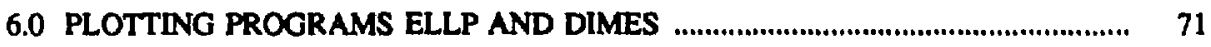

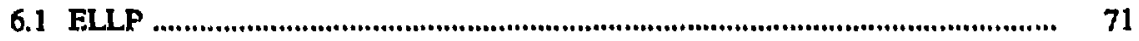

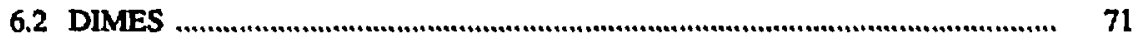

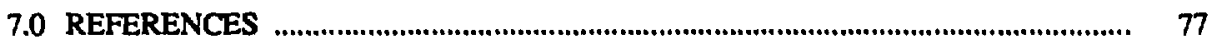




\section{List of Figures}

Figure 1.1. Programs for 2-D and 3-D modelling of now through fractured rocks. 3

Figure 2.1. Part of a fracture line inside the flow region. 15

Figure 2.2. Checking side numbers to determine if a fracture line passes through 16 the flow region.

Figure 2.3. Discarding fracture lines which are parallel to the boundaries.

Figure 2.4. Using array [ $t]$ to discard fractures which fall outside the flow region. 19

Figure 2.5. Truncation of fractures which fall both inside and outside the flow 20 region.

Figure 2.6. Boundary conditions applied to fracture models for permeability meas- 26 urement.

Figure 2.7. Distortion of isopotentials in an anisotropic medium with "no flow" 27 boundaries.

Figure 3.1. Two-dimensional fracture mesh. (a) fractures generated pseudo- 32 randomly, (b) same mesh with simple dead-ends removed.

Figure 3.2. Three-dimensional channelized fracture mesh. (a) random discs and 33 channels, (b) channels only.

Figure 3.3. Simple mesh with dead-ends highlighted.

Figure 3.4. Mesh from Figure 3.3 at the end of the first search. 38

Figure 3.5. Second sufficient condition. Respective positions of boundaries, anticu- 39 lation points and dead-end cluster.

Figure 3.6. Mesh from Figure 3.3 at the end of the first downward search.

Figure 3.7 Mesh from Figure 3.3, second loop of searches. (a) forward search, (b) downward search: only one source is left for the next forward search. The algorithm stops, nodes 8 and 9 are discarded.

Figure 3.8. Node renumbering, after Robinson (1982).

Figure 4.1. Two sets of boundary conditions for directional permeability. 
- viii -

Figure 4.2. A $70 \mathrm{~m}$ by $70 \mathrm{~m}$ flow region with six nested study regions.

55

Figure 4.3. Notations for computing the head at study region boundaries.

57

Figure 5-1. Flow regions with various orientations for directional permeability stu-

60 dies.

Figure 5-2. A set of directional permeability measurements plotted as $1 / \sqrt{\mathrm{K}_{\mathrm{g}}}$ in

62 polar coordinates.

Figure 5.3. A set of directional permeability measurements plotted in cartesian 64 coordinates

Figure 6.1. Polar and cartesian plots of directional permeabilitics.

72

Figure 6.2. Fractures in the generation region, and the $0^{\circ}$ notation flow region

74

Figure 6.3. Line network in flow regions.

75 
- ix -

\section{Acknowledgements}

The authors thank Lea Cox and John Peterson for providing a thorough review of this report. 


\subsection{INTRODUCTION}

Network models are useful tools for understanding the hydrology of fractured rock. Such studies of fracture hydrology can proceed by adopting a model for the network geometry, estimating the statistical distribution of the appropriate geometric parameters through field measurements, and generating realizations of statistically identical networks. Once the geometry of a particular realization is specifted. flow through the network can be studied (Long et al., 1982). For example, one might use such a procedure to study the avcrage equivalent permeability of a fracture network under various boundary conditions or as a function of scale of measurement (Long and Witherspoon, 1985). Such a study might be part of a larger study of hydrologic response to a perturbation such as the construction of an underground opening containing nuclear wastc.

The advantage of the network model approach is that one is able to configure tests of system behavior in any manner desired. For instance, if we wish to know how the rock behaves under a regional gradient which is approximately linear, we can impose a linear gradient on the model. If we want to know the behavior as a function of scale, we simply change the size of the model. In the ficld one is normally constrained to the approximately radial flow imposed by a well test, and it may be difficult to know what volume is actually being tested and how that volume is likely to behave under different boundary conditions. On the other hand, numerical models must make assumptions about the fractures which may be false. For instance, network models usually assume that flow occurs in the fractures as between parallel plates, although in some cases, channeling may dominate the flow. Also, it is difficult to obtain appropriate data for the network geomerry and especially the hydraulic conductivity of the individual fractures. Network modeling and in situ testing have complementary problems, and therefore the uses of these two techniques shouid be complementary. An appropriate phi- 
losophy is to improve the network model at least until one can explain the in situ test results with the model.

Besides constituting a complete chain of programs for the study of the equivalent permeability of two-dimensional fracture networks, FMG, RENUM, LINEL and ELLFMG are also part of a broader set of programs capable of modelling how and transport in both twodimensional and three-dimensional line networks (Figure 1.1). FMG3D, DISCEL and DIMES' three-dimensional capabilities are documented in Gilmour et al. (1986a and b). CHANGE is documented in Billaux and Long (1988a and b). TRINET is documented in Karasaki (1987). The complete set of programs as described in Figure 1.1 is a unique tool for the modelling of flow and transport in complex two- or three-dimensional fractured rock geometries. 


\section{FMG3D}

FMG

Generate 2-D

Line Fractures

$\%$

DIMES (2-D)

Plot 2-D Mesh

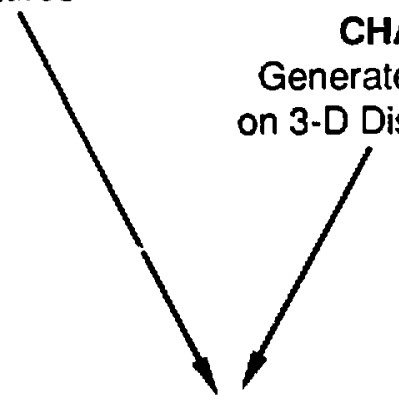

RENUM
CHANGE

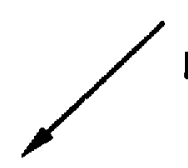
Generate 3-D Disc Fractures

|

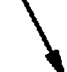

DIMES (3-D)

Plot 3-D Discs

Reorder and Simplify

2-D or 3-D Line Mesh
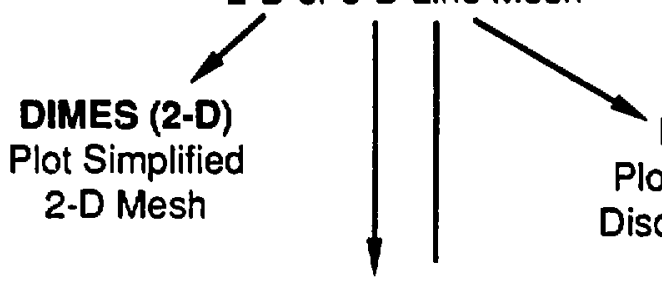

DIMES (3-D)

Plot 3-D Discs and Channels

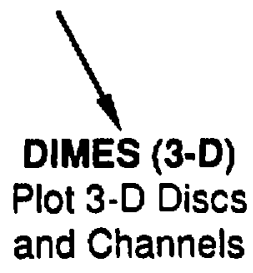

Plo: Simplified 3-D

Discs and Channels

\section{LINEL}

Compute Steady-Stale

Flow in 2-D or 3-D

Mesh

ELLFMG

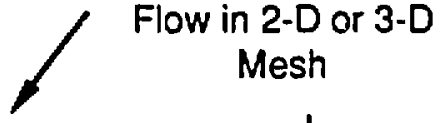

Compute Equivalent

Permeability of 2-D

Mesh

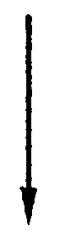

\section{TRINET}

Compute Transient Flow

$$
\text { and Transport in 2-D }
$$

or 3-D Mesh

\section{ELLP}

Plot 2-D

Permeability Ellipse

Figure 1.1. Programs for 2-D and 3-D modelling of flow through fractured rocks. 


\subsection{FRACTURE MESH GENERATION PROGRAM FMG}

The purpose of progrum FMG is to create a fracture network input file for the optimization progran, RENUM. To do this, FMG can either stant with a specified fracture system or generate a madom system based on stochastic variables provided by the user. By either process, the location, orientation, length, and aperture of all fractures in a generation region are determined. Next, FMO identifes only those fractures that are within subregion called the flow region and are connected by at leat one path to its boundaries. FMG determines the boundary nodes which are the inierections between fractures and fow region boundary lines and the interections between fractures, which are called intemal nodes. Non-conducting frictures may be eliminared from the fracture system. The geometric information needed to calculate flow is written into a file to be read by RENUM. RENUM will optimize the mesh. Flow through the fracture network of the flow region will be calculated by LINEL.

The first step is to generate a priniary fracture system within a rectangular or circular region called the generation region. The fracture system is generated using one or more fracture sets. The number of sets, the number of fractures per set and the dimensions of the generation region [xgene, ygene] or [rgene] are specified by the user in the file FMG.INP. The fracture characteristics (coordinates of fracture center, orientation, length and aperture) may either be specified by the user or stochastically generated on a set by set basis.

Distribution functions that can be used to generate fracture orientation angles, lengihs, and apertures currenlly includk: normal, lognormal, exponential, liniform, and nornal with an option to correlate fracture aperture to fracture length. Fracture lengths are usually allowed to vary lognomally. The fracture apertures are usually generated assuming that apertures are lognormally distribured within a set. In general, field data will dictate which distribution function is appropriate for each fracture characteristic. The fractures extending beyond the bosundary of 
the generation region are trunceted.

There are two options for stochastically generating the fracture mesh. In the first option ([nsgene] $]=$ ) the fracture network is generated set by set for the entire generation region such that the fracture centers are randomly located and uniformly distributed throughout it. In the second option ([nsgene]>0) the generation region is divided into a number of square or rectangle subregions, and the generation of the fracture mesh is made by subregions. In this case the fracture characteristics are elther read in or senerated for the first set and each subregion, second set and each subregion and 20 on, until the number of sets [nsets] is exhausted. The fracture characteristics are stored in the array $[\mathrm{frac}(\mathrm{nfrc}, 10)]$ to be used in later computations. The option of dividing the generation region into wubregions and generating the fracture network by sub. agions was introduced to obtain a heterogeneous (i.e. "spatially variable") distribution of the mesh characteristics in the generation region.

Next, rectangular or circular thow region is defined within the generation region. Input parameters that define the flow region include the two side lengths [xmesh, ymesh] and the orientation angle $(\theta)$, or the radius of the flow region [mesh]. A circular hole can also be specified when a circular flow region is used. Its center [xhole, yhole] and radius [thole] are specified by the user. The flow region is centered in the generation region. For a given primary fracture system, any number of flow regions of varying size and orientation or with different holes, may be defined and corresponding flow meshes generated.

The system of fractures lying within the flow region is determined by comparing the position of each fracture with the position of the four boundary lines or with the position of the flow and hole boundary circles. Fractures lying entirely inside the flow region are saved and fractures lying entirely outside the flow region are discarded. Fractures intersecting boundary lines are saved and the intersection(s) are stored as boundary nodes. A fracture may intersect more than one boundary line (depending on the geometry of the flow region, fracture location and length) and, therefore, contain more than one boundary node. Fracture lengths are truncated at boundary lines, and only the part falling inside the flow region and outside the hole (if 
any) is used to solve for flow in the fracture network.

The system of fractures contributing to flow through the region is determined next. Intersections between fractures are found and stored as intemal nodes and the segment between two consecutive nodes is defir ad as an element. Flow in a fracture can take place only through the elements of the fracture network so that in determining the fracture network for a steady state computation, any fracture containing less than two nodes cannot conduct flow and may, therefore, be discanded from the system of conducting fractures. The program starts with the fractures thas intersect the flow region boundaries and determines the fractures they intersect. Then It considers the fractures it has just found and looks for new fractures intersecting them. This process goes on until there are no more fnctures to be checked. As mentioned at each level the non-conducting fractures are discanded.

Finally, flow network information is prepared and written for input to the mesh optimization code, RENUM. Nodal arnys are assembled from stored boundary and intemal nodes on conducting fractures in the flow region. All necessary run identification, fracture system, flow region, and nodal data are written to a file. This section describes the program FMG. The user's guide for the program as well as a listing of the code are provided in a separate report.

\subsection{Generation of Fracture System}

A primary fracture system, consisting of one or more sets of linear fractures randomly distributed within a rectangular or circular generation region, is created and fractures extending beyond the boundary are truncated.

\subsubsection{Fracture System Characteristics}

In the stochastic mode the primary fracture system is generated using parameters specified by the user. These include:

- the dimensions of the rectangular generation region, $x_{2}$ and $y_{2}$ ([xgene],[ygene]), or $r_{2}$ ([rgene])

- the number of fracture sets [nsets], 
- either the number of fractures per set [nfrac] or the fracture density (i.e., number of fractures per unit area) per set [rlamb],

- distribution parameters for generating fracture orientation angles, lengths, and apertures.

The fracture centers are randomly generaled within the region. The main program, FMG, calls subroutine FRAGEN to read input parameters and coordinate the generation of fracture characteristics. This section describes how FRAGEN works in the stochastic mode. Altematively fracture centers, orientation angles, lengths, and apertures can be specified for any or all fracture sets.

Fracture Centers. The fracture centers are generated by either of two subroutines RECTXY and CIRCXY.

Sutroutine RANDXY generates centers randomly distributed throughout a rectangular region. Coordinates of fracture centers $\left(x_{c}, y_{c}\right)$ are computed by generating doublets of random numbers, uniformly distributed between zero and one, then scaling them by multiplying by the length of the generation region [xgene] ([ygene]), and suburacting $1 / 2$ [xgene] (1/2 [ygene]). This makes the center of the generation region the origin of the rectangular coordinate system.

Subroutine CIRCXY generates centers randomly distributed throughout a circular region. The coordinates $\left(x_{c}, y_{d}\right)$ are computed by generating doublets of random numbers $(a, b)$, uniformly distributed between zero and one, subtracting 0.5 from them, and then keeping only the pairs of numbers such that

$$
a^{2}+b^{2} \leq 0.25
$$

$a$ and $b$ are then scaled by multiplying them by twice the radius of the generation region $2 *$ rgene].

Orientation. The orientation of each fracture line is determined by the angle which the fracture forms with the $x$-axis of the generation region. Fracture orientation angles are either input or generated by the program. If statistically generated, the mean orientation of each fracture set if required as well as other parameters, depending on the distribution chosen. 
Fracture Lengths and Apertures. The length and aperture of each fracture are generated according to a normal, lognomal or exponential distribution. The generation procedure for the first two distributions requires the mean [ev] and standard deviation [sd] be specified for each fracture set. The exponential distribution requires only the mean. Apertures may also be correlated with fracture lengths (see the use of subroutine NORMD1).

\section{Statistical Simulation}

Random number generator. The statistical distribution subroutines and RANDXY use a random number generator called GGUBFS which is an International Mathematical and Statistical Library (IMSL) subroutine. GGUBFS retums random numbers uniformly distribulnd between zero and one and requires a double precision seed value [dseed]. GGUBFS retums a different random number each time it is called within a program. However the same sequence of random numbers is produced each time the program is run with the same initial seed. This mode of operation is optionally overridden by generating an arbitrary seed,

$$
\text { dseed }=\operatorname{SECNDS}(0.0) * 100.0
$$

where SECNDS is a VAX-11 FORTRAN function subprogram which retums the system time of day in seconds less the value of its argument. An input flag [iranf] controls whether the seed is read or generated, and the initial seed is printed out. Since the seed defines the starting location for the random number generator, the user can reproduce a series of random numbers, i.e., reproduce a random fracture system by inputting the same initial seed in 2 later run.

Random generation of fracture centers. Depending upon the value of the input parameter [igene], subroutine RECTXY or CIRCXY calls GCUBFS once for each coordinate of the fracture center, to get two numbers $a$ and $b$. The coordinates are then computed from a and $b$ using either:

$$
\begin{aligned}
& \left.x_{c}=\text { FLOAT[INT }\left(x_{1} * 10^{n} * a\right)\right] / 10^{n}-x_{8} / 2 \\
& \left.y_{c}=\text { FLOAT[INT }\left(y_{z} * 10^{n} * b\right)\right] / 10^{n}-y_{2} / 2
\end{aligned}
$$

in the case of a rectangular generation region, or

$$
x_{c}=\operatorname{FLOAT}\left[\mathrm{NTT}\left(2 \cdot \mathrm{rg} \cdot 10^{n} *(\mathrm{a}-0.5)\right] / 10^{n}\right.
$$




$$
y_{c}=\operatorname{FLOAT}\left[\operatorname{INT}\left(2 \cdot \mathrm{rg} \cdot 10^{\mathrm{n}} *(\mathrm{~b}-0.5)\right)\right] / 10^{n}
$$

in the case of a circular generation region. In the latter case, a pair $(a, b)$ is used only if

$$
a^{2}+b^{2} \leq 0.25
$$

otherwise, $a$ and $b$ are discarded and GGUBFS is called again twice to get a new pair. In the equations above, $n$ is the number of decimal places in the coordinate [itole], $x_{g}$ is the length of the generation region in the $x$ direction [xgene], $y_{g}$ is the length of the generation region in the $y$ dinection [ygene], $\mathrm{rg}$ is the radius of une generation region [rgene], and $\mathrm{d}$ [dseed] is a double precision dummy variable initially equal to the input or generated seed then reset by GGUBFS. NNT and FLOAT are intrinsic library functions which convert a real number to an integer by truncation and an integer to a real number, respectivcly. Truncating coordinates to $\mathrm{n}$ decimal places limits the minimum distance between fracture centers to the value $10^{-n}$.

\section{Random Generation of Fracture Characteristics}

Normal distribution. In subroutine NORMAD, the sum $S_{\mathrm{N}}$ is calculated by calling GGUBFS twenty-five times and accumulating the sum,

$$
S_{N}=\sum_{n=1}^{25} r_{n}
$$

where $r_{n}$ equals the value retumed by a call to GGUBFS, $r_{n}=$ GGUBFS(d). As shown by Hammersly and Hanscomb (1964), $\mathrm{S}_{\mathrm{N}}$ is distributed normally with an expected value of $25 / 2$ and a variance of $12 / 25$; therelore,

$$
S_{N}^{*}=\sqrt{\frac{25}{12}} S_{N}-\frac{25}{2}
$$

is distributed normally with expected value 0 and variance 1 . If $\mu$ and $\sigma$ are the expected value and standard deviation supplied by the user ([ev] and [sd]) then

$$
x=\sigma S_{N}^{*}+\mu
$$

is distributed normally, $N(\mu, \sigma)$ with the specified parameters. (Note that in this equation, $x$ does not refer to a point coordinate.)

Lognormal distribution. If $S_{\mathrm{N}}^{*}$ and $\mathrm{x}$ are defined as in the previous section, then $S_{\mathrm{N}}^{*}$ is distributed $N(0,1)$, and $x$ is distributed $N(\mu, \sigma)$, and $y=\exp (x)$ is distributed lognormally. 
In terms of the parameters $\mu$ and $\sigma$ of the nomal distribution for $x$, the mean $\alpha$ and variance $\beta^{2}$ of the lognormal distribution are

$$
\alpha=\exp (\mu) \exp \left(\sigma^{2} / 2\right)
$$

and

$$
\beta^{2}=\exp \left(\sigma^{2}+2 \mu\right)\left[\exp \left(\sigma^{2}\right)-1\right] \text {. }
$$

Since the user will specify $\alpha$ and $\beta$, it is necessary to solve for $\mu$ and $\sigma$ in terms of these variables:

$$
\begin{gathered}
\mu=2 \ln \alpha-\frac{1}{2} \ln \left(\beta^{2}+\alpha^{2}\right), \\
\sigma=\sqrt{\ln \left(\beta^{2}+\alpha^{2}\right)-2 \ln \alpha}
\end{gathered}
$$

Therefore, subroutine LOONOD can calculate y from

$$
y=\exp \left(\sigma S_{N}^{*}+\mu\right) \text {, }
$$

where $\mu$ and $\sigma$ are defined above and $\alpha$ and $\beta$ are specified by the user ([ev] and [sd]).

Exponential distribution. In subroutine EXPOND, $\mu$ is the expected value given by the user [ev] and

$$
x=\mu \ln (1-r)
$$

is distributed exponentially, where $\mathrm{r}=$ GGUBFS(d).

Normal distribution correlating two variables. Subroutine NORMD1 generates random variables, $x$, distributed normally where the expected value, $\mu$, is proportional to the logarithm of another parameter, $x_{1}$ or to the parameter itself. This correlation may be used to compute fracture aperture as a function of fracture length. $S_{N}^{*}$ is defined as before (Equation 2-5) and the standard deviation, $\sigma$, is supplied by the user as [sd]. The user also supplies the $y$ intercept and the slope ([ycept] and [slope]) of a linear relationship between mean values of the

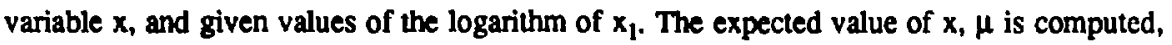

$$
\mu=\text { ycept }+ \text { slope } * \log _{10}\left(x_{1}\right)
$$

and $x$ is computed as before, (Equation 2-6).

Uniform distribution. Subroutine UNIFOD generates random variables, $a_{i}$, distri- 
buted uniformly over a given range, ainf to asup, such that:

$$
a_{i}=(\text { asup }- \text { ainf })^{* G G U B F S(d s e e d)}+\text { ainf }
$$

Additional distributions. The fracture gencration code can easily ue miouified to include additional distribution functions that are found to be appropriate for any of the fracture characteristics.

\subsubsection{Equations of Fracture Lines}

The coefficients of the equation of the line in which a fracture lies are computed by submutine EQLINE from the fracture's orientation and the coordinates of the fracture's center. The equation of a line is $a x+b y+c=0$, where $a=-\sin ($ orie); $b=\cos ($ orie) and $c=-b * y c$ - a * xc are the coefficients of the line with a given orientation [orie] and passing through the fracture center of coondinates (xc,yc).

\subsubsection{Truncation at Boundsry}

Fractures extending beyond the generation region are truncated at the boundary by the subroutine LIMIT. If a fracture is truncated the coordinates of the end points and the length of the fracture are recalculated. The fractures lying inside the generation region are used for statistical calculations and to determine the flow for a given study region.

\subsubsection{Statistical Calculations}

At the end of the generation stage simple statistical calculations are performed. This task is done by the subroutine PFS which calculates and prints the fracture statistics elements ([sd] and [ev]) for orientation, length and aperture in order to compare the generated fracture system with the inpul parameters provided by the user.

These statistics are computed two other times in the program, when the fracture network is altered. 


\subsection{Fracture System in the Flow Region}

The flow region, which is the part of the fracture network to be analyzed, is included in the generation region and only fractures within this region are considered for input to the fluid flow model. For one random generation of fractures, several flow regions can be specified. They will be processed sequentially, and a finite-element mesh will be built for each of them. Fractures extending beyond the flow region are truncated at the boundary and those lying entirely outside the region are discarded. Intersections between fractures and flow region boundary lines are identified at this time. These intersection points, called boundary nodes, will be assigned fixed head or fixed flux in subroutine WRENUM.

\subsubsection{Flow Region}

The flow region is either defined by a rectangle sentered at the origin of the generation region ([igene] $=0$ ), or defined by a circular outer boundary and a circular innerhole ([igene] = 1). The dimensions, $: x m e s h]$, [ymesh], and the orientation angle $\theta$ [theta] of the rectangle, or the radius [mesh] of the outer boundary and the radius [mole] and position [xhole, yhole] of the hole are input parameters read from FMG.INP. They must be such that the resulting region lies completely within the generation region. The next step is to determine which fractures of the primary fracture system lie within the flow region.

\subsubsection{Fractures in Flow Region - Rectangular Case}

Subroutine RLIMIT truncates the fractures intersecting the rectangular flow region boundaries and discards those falling completely outside of it. If a certain fracture is truncated, the subroutine RLIMIT will recalculate the coordinates of the end points and the length of the truncated fracture. The information thus obtained about fractures falling within the flow region will be stored and used to determine the flow mesh.

We mentioned that the flow region is centered in the generation region. Then the equations of the boundary lines will be:

$$
A x+B y \pm C=0
$$




$$
B x-A y \pm D=0
$$

where $C=y m e s h / 2$ and $D=x m e s h / 2$.

Using the equation of the line that passes through each fracture or "fracture line", we then determine its intersection with the four lines that lie along the flow region boundaries. Using the coordinates of the first endpoint of the fracture, the two or four intersections are parameterized using the parameter $t[t(4)]$ such that:

$t=0$ at the first endpoint of the fracture

$t=$ length of the fracture at the other endpoint

The number of intersections, either 2 or 4 , is stored in variable [nt]. From Figure 2.1, one can see that the part of the fracture that falls inside the flow region has to lie between the two middle intersections. The array [t] is ordered. Then in order to determine if the fracture line passes through the flow region, the first two values in the ordered [t] array are considered. If they correspond to two adjacent sides, then the fracture line passes through the flow region. If they correspond to two opposite sides, then the fracture line does not intersect the flow region (Figure 2.2), except in the special case of the fracture being parallel to boundaries In this case, the fracture is discarded only if its distance to the center is greater than the relevant flow region size [xmesh]/2 or [ymesh]/2 (Figure 2.3 ). Since the sides of the flow regioil are numbered consecutively, the sum of the numbers of two adjacent sides is odd, whereas the sum of the number of two opposite sides is even. The side numbers for each intersection are stored in the array [is(4)]. So we want to keep all fracture lines with four intersections (i.e. non-parallel to a side) and an odd sum is $(1)+$ is(2), and all fracture lines with two intersections (i.e. parallel to a side). This is achieved by keeping only fracture lines for which the sum [is(1) + is(2) $+n t / 2]$ is odd.

At this point we have determined if a fracture line passes through the flow region. We also know which segment of the fracture line is in the flow region. It is the segment $t 1-t 2$, with $t 1=[t(1)]$ and $t 2=[t(2)]$ when $[n t]=2$, and with $t 1=[t(2)]$ and $t 2=[t(3)]$ when $[n t]=$ 4. We must now determine which part of the fracture overlaps this segment. If all the fracture 


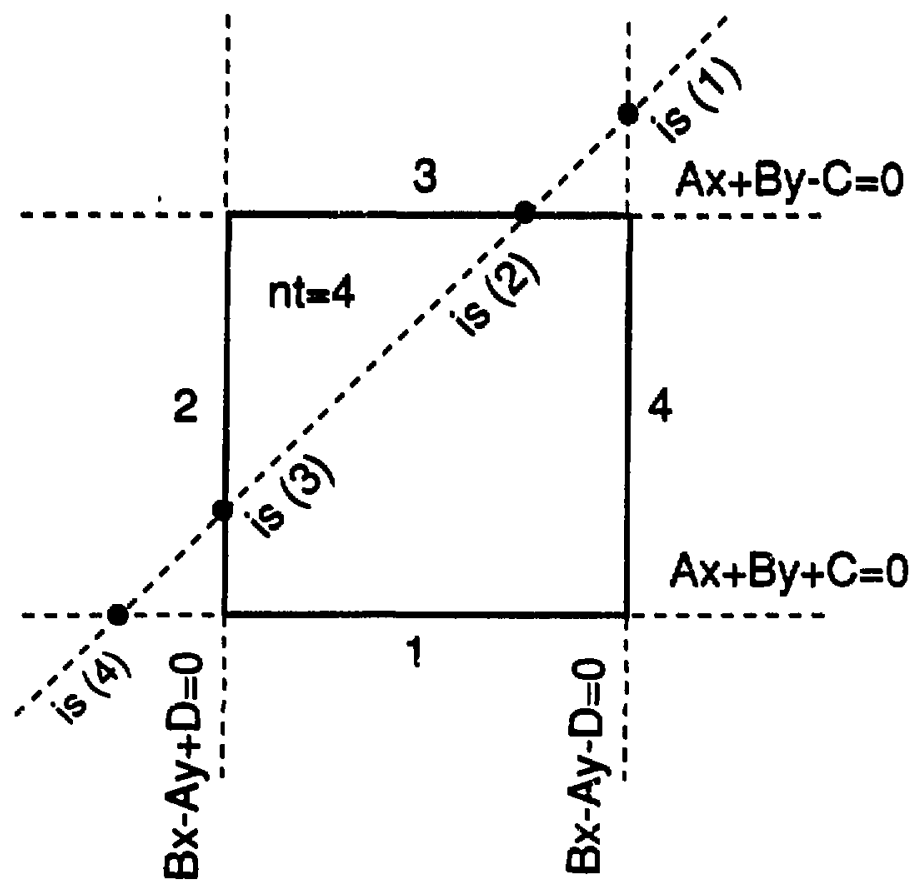

Figure 2.1. Part of a fracture line inside the flow region. 


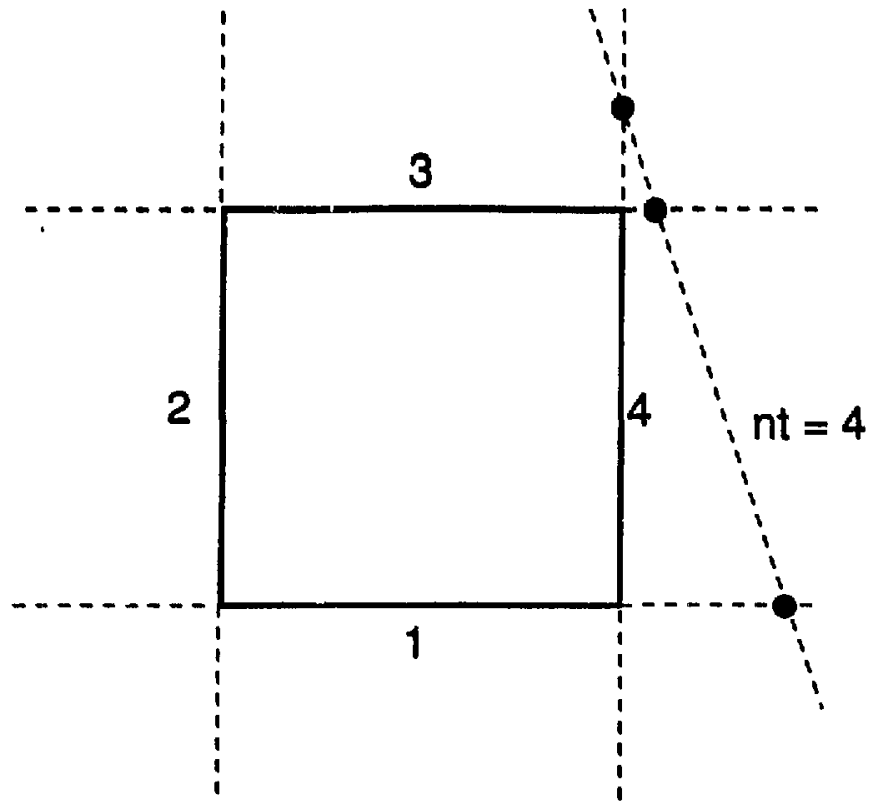

Figure 2.2. Checking side numbers to determine if a fracture line passes through the flow region. 


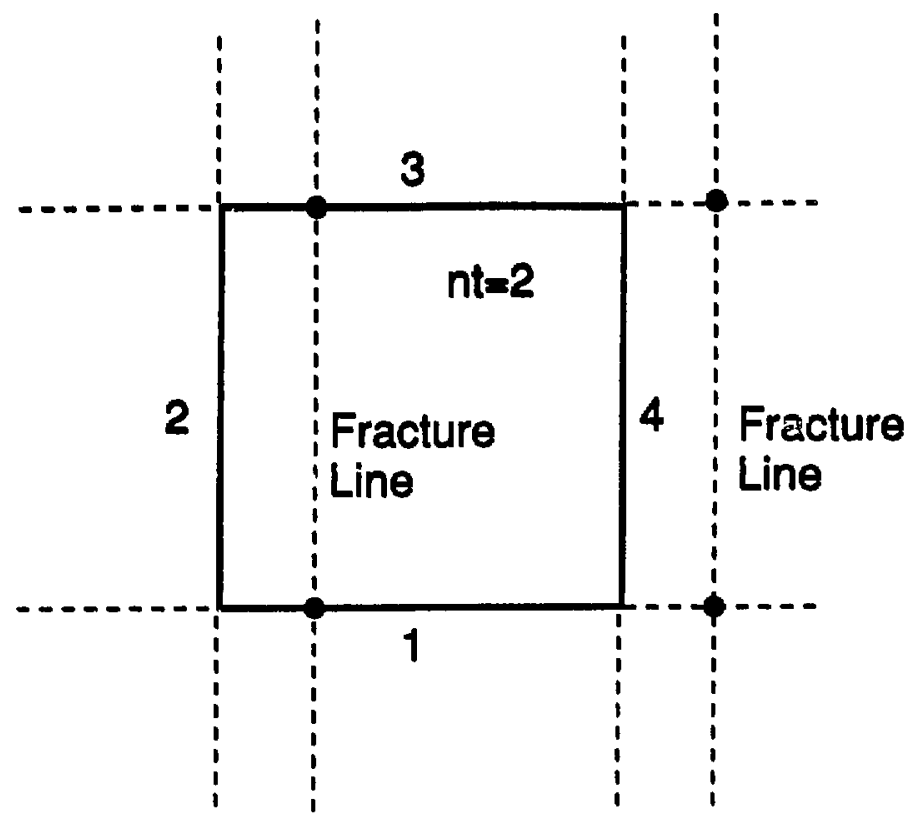

XBL 882-10059

Figure 2.3. Discarding fracture !ines which are parallel to the boundaries. 
is inside the segment, we keep it. If only part of the fracture is inside the segment, we truncate this fracture. Finally, if the fracture and the segment do not overlap, we discard the fracture. If $\mathrm{tl}>$ fracture length or $\mathbf{2}<0$ (Figure 2.4) the fracture is outside the flow region. Otherwise, the fracture is kept, and truncated if needed (Figure 2.5). The new fracture information is stored in array [frac].

\subsubsection{Fractures In Fow Reglon - Circular Case}

Subroutine CLIMIT truncates the fractures intersecting the circular flow region boundaries and discands those falling completely outside of it. If a certain fracture is truncated, the subroutine CLIMIT will recalculate the coordinates of its endpoints and the length of the truncated fracture. A fracture may also be split in two parts by the inner hole. In this case, two fractures with the proper endpoints and lengths are created from the generated one. The information thus obtained about fractures falling within the flow region is stored and used to determine the flow mesh. A do loop nver the generated fractures is performed. After its proper endpoints and length have been recomputed to account for eventual truncation, the characteristics of a fracture are rewritten in the same array [frac]. However, the identification number of the fracture (i.e. its position in the array [frac]) may change after it has been examined, because whenever a fracture must be discarded, the counter for writing characteristics into [frac] is not incremented, so that the next fracture will be written over it in the array.

First the fracture is truncaied at the perimeter of the outer flow region circle. Since the nrigin of the coordinates is its center, the equation of the flow region circle is:

$$
x^{2}+y^{2}=r_{m}^{2}
$$

where $r_{m}[m e s h]$ is the radius of the circle. We parameterize the line supporting the fracture by the relative distance $t$ from the first endpoint $(x, y)$ :

$$
\begin{aligned}
& t=0 \text { at the first endpoint }\left(x_{1}, y_{1}\right) \\
& t=1 \text { at the second endpoint }\left(x_{2}, y_{2}\right)
\end{aligned}
$$

then, if alen is the length of the fracture, the distances $t 1$ and 2 for the intersections of the line 

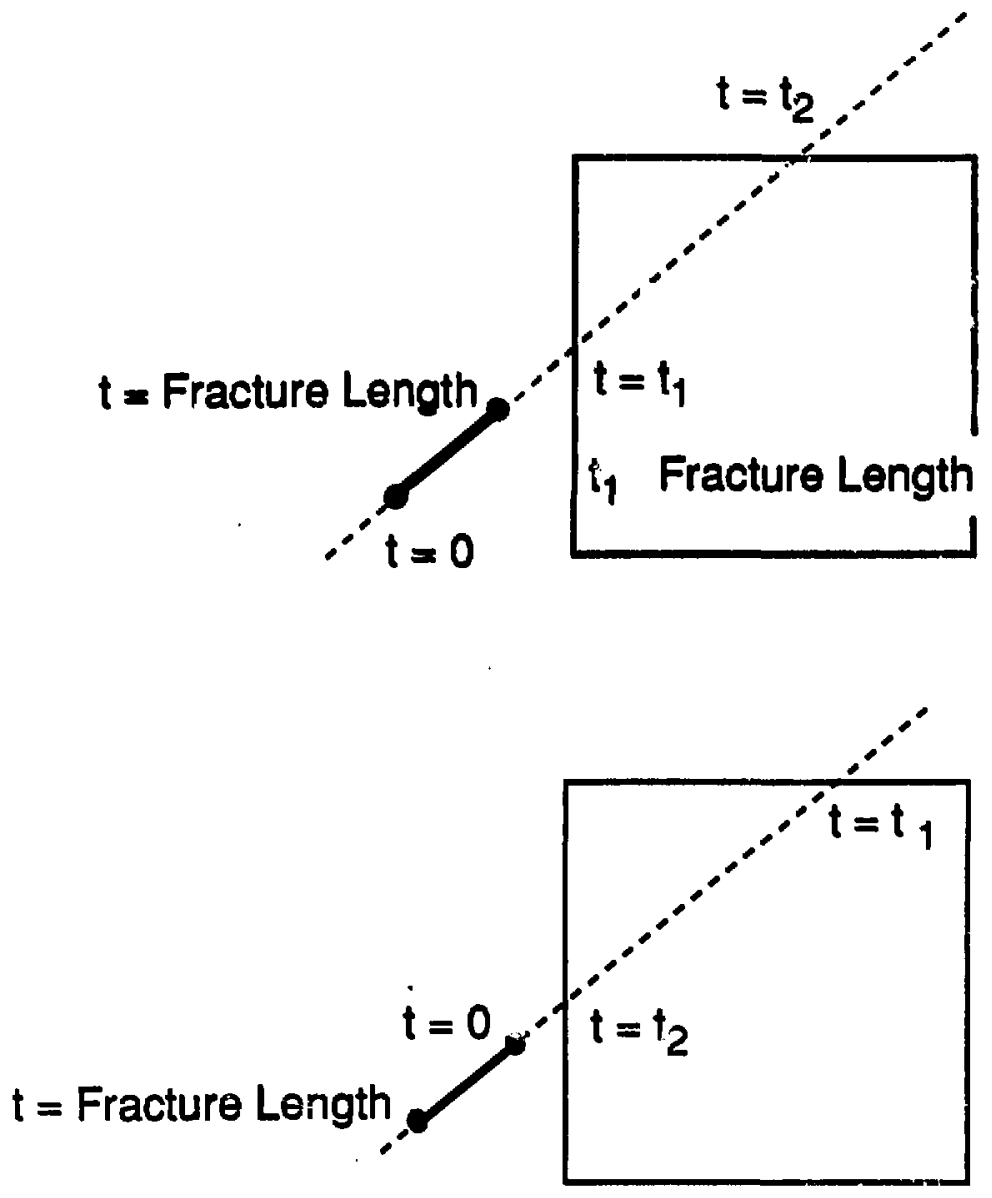

Figure 2.4. Using array [t] to discard fractures which fall outside the flow region. 

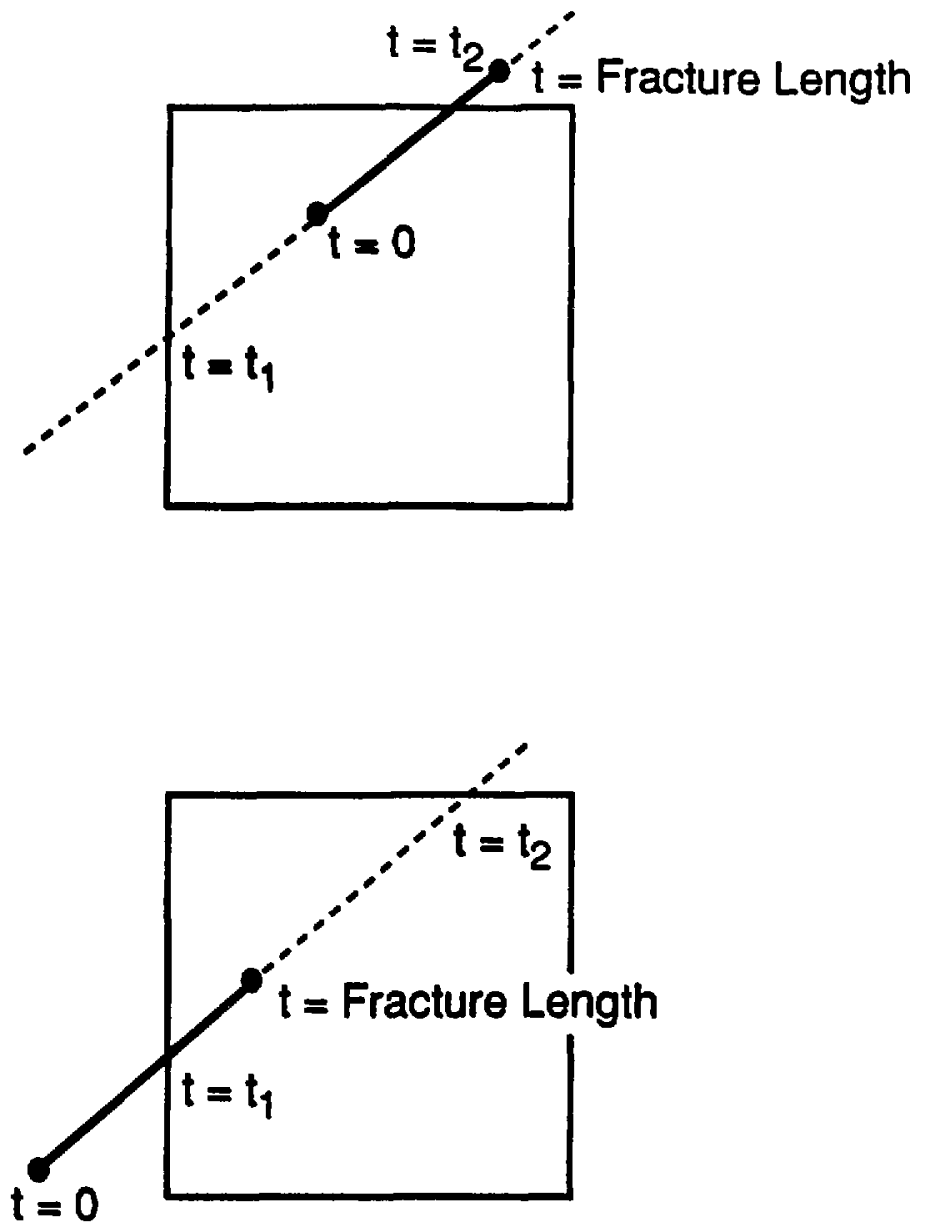

XBL 882-10061

Figure 2.5. Truncation of fractures which fall both inside and outside the flow region. 
with the circle are the solutions of:

$$
\text { alen } t^{2}+2\left[x_{1}\left(x_{2}-x_{1}\right)+y_{1}\left(y_{2}-y_{1}\right)\right] t+x_{1}^{2}+y_{1}^{2}-r_{m}^{2}=0
$$

The fracture is at least party inside the flow region if

- the discriminart of equation 2-13 is positive (i.e. the fracture line cuts the flow region)

- t1 is less than 1,

- $\mathbf{2}$ is more than 0 .

Otherwise it is diecanded.

The first endpoint is truncated if $t 1$ is greater than zero. The second endpoint is truncaled if $t 2$ is smaller than one. The values of $x_{1}, y_{1}, x_{2}, y_{2}$ and alen are modified accordingly if necessary.

Next the fracture is truncated or split at the inner circle, or "hole", with radius th[rhole], and center $\left(x_{h}, y_{h}\right)$ [xhole, yhole]. The coordinates of the endpoints are translated

$$
\begin{aligned}
& x x_{1}=x_{1}-x h \\
& y y_{1}=y_{1}-y h \\
& x x_{2}=x_{2}-x h \\
& y y_{2}=y_{2}-y h
\end{aligned}
$$

Then the equation for the intersection(s) is exactly similar to equation 2-13:

$$
\text { alen }{ }^{2} t^{2}+2\left(x x_{1}\left(x x_{2}-x x_{1}\right)+y y_{1}\left(y y_{2}-y y_{1) 1} t+x x_{1}^{2}+y y_{1}^{2}-m^{2}=0\right.\right.
$$

This time we wank to discard anything inside the circle.

So a fracture will be kept unchanged at this step if:

- the discriminant of equation 2-14 is negative (t.c. the fracture line does not cut the cincle)

- $t 1$ is more than 1

- 2 is less than 0 
A fracture, will be discarded if $t 1$ is less than zero and 2 is more than one (fracture totally included in the hole). If either $\mathrm{t} 1$ is more than zero or $\mathrm{t} 2$ is less than one, then the second endpoint or the first endpoint respectively is truncated, so the endpoint coordinates and length are updated. If both tl and $t 2$ are between zero and one, then the fracture must be split in two fractures. This is done by simply assigning to the current fracture number the first part of the fracture, and then by considering the second part of the same fracture, instead of the next fracture, when incrementing the fracture number. This requires that the number of the next gencrated fracture to be processed be higher than the number of the current truncated fracture plus one. This may be true because some previous fructures have been discarded. If it is not true, subroutine MOVE is called, and translates all the characteristics of the fractures not yet studied down in the array [frac]. The amount [idif] by which the characteristics are translated down is determined by [idif] $=$ minimum $([n f r a c] / 10+1$, [maxfrc] $-[$ [nfrac]), where [nfrac] is the number of fractures, and [maxfrc] is the size of the array [frac].

\subsubsection{Statistical Calculations}

After eliminating all fractures outside the flow region, and truncating all those which intersect the flow region boundaries, the same statistics as described in Section 2.1.4 are computed and printed by subroutine PFS. Comparison of these values with those computed for the generation region indicates whether the flow region is statistically representative.

\subsubsection{Connections between Sides}

When studying the percolation properies of networks, one important parameter is the percolation threshold. This is the "minimum" set of statistical properties for which a connected network of infinite size (infinite cluster) exists. Below the threshold, only finite-size clusters of fractures exist. In order to compute this threshold, we generate large networks and simply check if there is a connection between sides 1 an 3 (rectangle), or between the center hole and the outer boundary (circle). The ability to check connections this way without outputting a finite element network has been added to the program. This is performed by subroutine CON- 
NEC if the flag [icont] is set to 5 in the input desk.

\subsection{Fracture System to be Used in Flow Model}

In order to calculake flow through the system, all fracture intersections must be located. Intersections between fractures and boundary lines have already been determined. Therefore, the next step is to locale all intersections between fractures (intemal nodes). This is done sequentially, stanting with fractures intersecting the boundaries, then fractures intersecting these ones, and so on, untl no new intersection is found. In this way, all the fractures from which there exists no path to any boundary, l.e. isolated clusters, are automatically discarded. In the circular case, the search for intersections is initulized with only the fractures intersecting the hole. In this way, only fractures linked to the hole will be kept. They are the only ones having an effect when modelling a well test. For steady state problems, flow in a fracture can only occur between intersections. Therefore, only those fractures containing two or more nodes may conduct flow. To simplify the flow problem, all fractures containing less than two nodes are identified as nonconducting and eliminated from the catalogue. The elimination of deadends and isolated clusters can both be overridden to produce meshes for transient or fracturematrix flow.

\subsubsection{Calculation of Fracture Intersections}

The fracture mesh is buill by subroutine CONNEC from the rectangular boundaries to the inside of the flow region, or from the inner hole to the outside, level by level. Intersections of all the fractures intersecting the boundaries (fractures in level 1) with all the fractures, either in level 1 or not, are searched. All the fractures not in level one but which intursect a fracture in level one are put in level two, and are then screened for intersections, and so on. Fracture numbers are rearranged level by level during the process, by permutating elements of the [iold] reference array pointing from rearranged numbers to original numbers. When a fracture [ifrac] is studied, only fractures with numbers bigger than [ifrac] are screened for intersections. If the fractures intersect, the coordinates of the point of intersection are determined. 
The following intersection information is stored: the identification numbers of intersecting fractures [ifrac(2,mnod)]; the distance from the first end point of the respective fracture [tint(2,mnod)]; the node numbers for each fracture [knode(mkey)]; the key to array [knode(mkey)] for each fracture [kut(mfrc)].

\subsubsection{Elimination of Nonconducting Fractures}

Depending on the value of the fiag [ikeep] input by the user, non-conducting fractures are discarded or kept in. If [ikeep] = 0 , simple dead-ends are discarded as well as isolated clusters. If [ikeep] $=1$, dead-ends are kept but isolated clusters are still discarded. If [ikeep] $=2$, then both dead-ends and isolated clusters are kept.

\section{Discarding simple dead-ends [lkeep] $=0$}

Fractures with only one intersection ane dead-ends for steady state flow. So at each level, once all the intersections of a given fracture have been found, if there is only one intersection, the fracture inay be eliminated. Note that by removing the fracture, we are removing one intersection from a fracture in the previous level. This other fracture may then be left with only one intersection, and so on. The program goes back through the mesh deleting fractures until it finds a fracture left with two or more intersections. Note that this fails to get rid of dead-ends containing loops. These complex dead-ends will be discarded by RENUM.

\section{Keeping isolated clusters [ikeep] $=2$}

Isolated clusters are discarded "by default" since the search for intersections is started at the boundaries and follows the connections. If [ikeep] $=2$, when the search initiated at the boundaries is finished, the number of fractures already screened is tested against the total number of fractures in the flow region. If they are not equal, a fracture not yet included is just put arbitrarily in the next level, and the search is resumed starting from that fracture. The process is then repeated until all the fractures in the flow region have been searched. 


\subsubsection{Boundary Conditions for Rectangular Networks}

The rectangular fracture networks produced by FMG are primarily used to compute directional conductivities. Such conductivities in several directions are then used to compute an equivalent co:2tinuous medium conductivity tensor, and to check if the continuum hypothesis holds by deriving a normalized mean square error. This is performed by program ELLFMG (Chapter 5). In a heterogeneous medium such as fractured rock, conductivity must be measured in the direction of the gradient. The average gradient can be constant in magnitude and direction throughout a heterogeneous region in steady flow if the region behaves like a homogeneous porous medium. The direction of flow, however, is controlled by the direction of the fractures. Since the direction of the gradient can be controlled, measuring permeability in the direction of the gradient is much easier than measuring in the direction of flow.

The boundary conditions necessary to produce a constant gradient in a rectangular anisotropic flow region are illustrated in Figure 2-6. They consist of two constant-head boundaries ( $\phi_{2}$ and $\phi_{4}$ ) and two boundaries with the same linear variation in head from $\phi_{1}=1.0$ to $\phi_{4}=0$. Conductivity is measured in the direction perpendicular to sides 2 and 4 .

The linearly varying boundary conditions in sides 1 and 3 are necessary because, in general, the medium in the flow region is anisotropic. Without these boundaries, the lines of constant head would be distorted near sides 2 and 4 as shown in Figure 2-7. When the isopotentiais are distorted, only part of the flow region can experience a constant gradient. In an arbitrary heterogeneous system of unknown anisotropy, it is impossible to determine which part of the system is experiencing a constant gradient and which part is not. Therefore when no flow boundaries are used, it is not always possible to measure only that part of the flux which is due to a known constant gradient.

The boundary conditions used in Figure 2.6 insure that the whole fracture system is equally stressed by the hydraulic gradient. Under these boundary conditions, the existence of a constant gradient in the flow region depends only on how well the fracture system is interconnected. If the system is well connected, it will behave like a porous medium and have a 


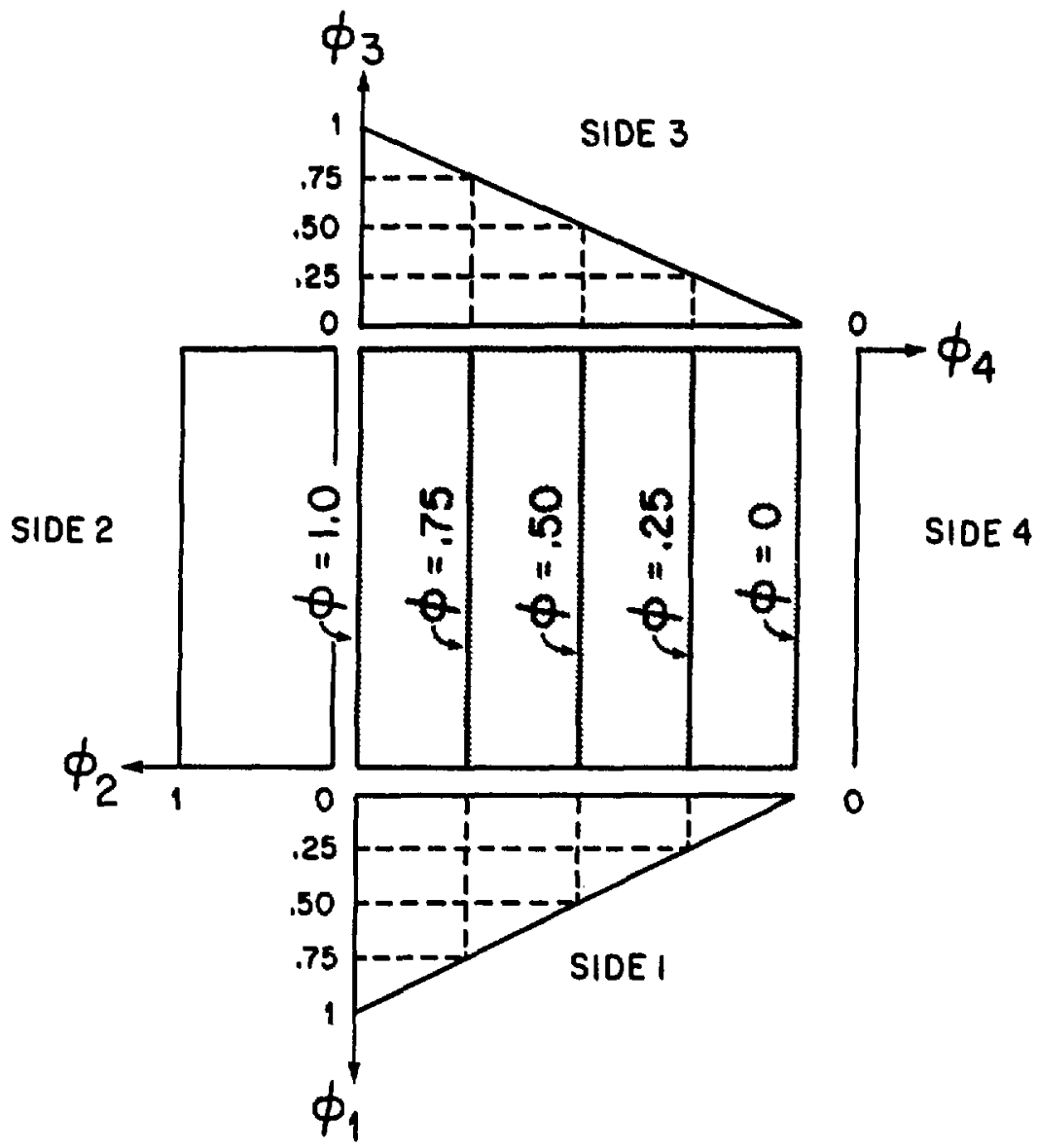

XBL 8010-2853

Figure 2.6. Boundary conditions applied to fracture models for permeability measurement. 


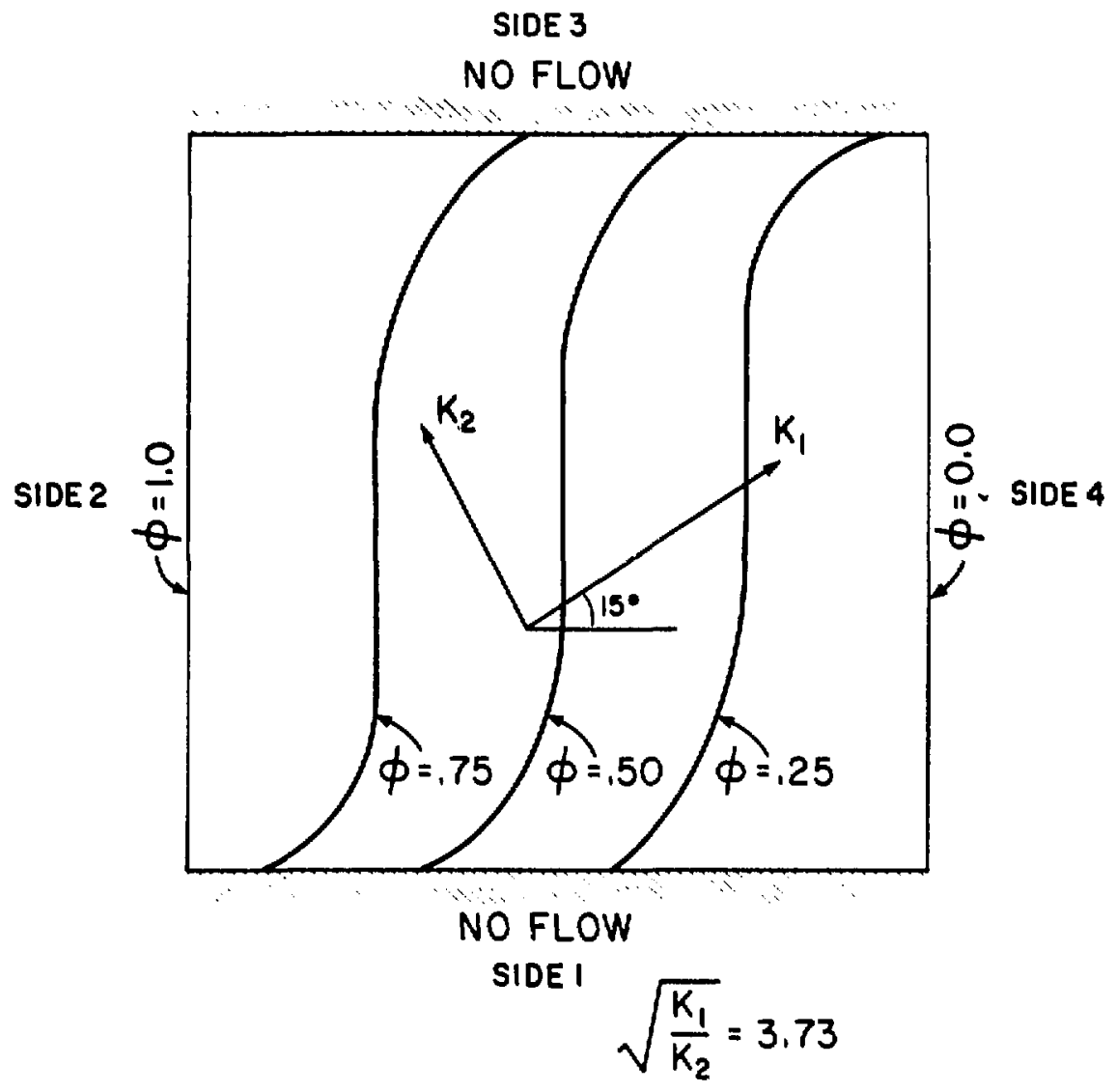

$X 8\llcorner 8010-2851$

Figure 2.7. Distortion of isopotentials in an anisotropic medium with "no flow" boundaries. 
constant gradient.

In order to accommodate directional conductivity studies, FMG can handle both constant and linearly varying imposed head boundary conditions. An option is also provided to produce constant flux boundary conditions. Note that if such conditions are used, the precessing will end with program LINEL (Chapter 4), and program ELLFMG will not be able to compute an equivalent porous medium conductivity tensor.

\subsubsection{Boundary Conditions for Circular Networks}

The circular networks produced by FMG are primarily used to simulate well-tests. A constant flux or constant head is imposed on the inner hole representing the well, and constant head conditions are imposed at the perimeter of the flow region. This reproduces the conditions during a well test.

\subsubsection{Finite Element Mesh}

Subroutine WRENUM reads the boundary conditions specifications for a given flow region and computes the imposed head at each boundary intersection if a linearly varying imposed head is specified. The following specifications for each node are then outpul:

- the identification number of the node

- a code identifying if the node is intemal, or a boundary with imposed head, or a boundary with imposed flux $(0,1,-1)$

- the side nurniver for a toundary node $(1,2,3,4$ if rectangular, 1,3 if circular)

- the coordinates of the node

- the value of the imposed head(s) or flux(es) at the node if needed

Finally, the element catalogue is printed, including:

- the element identification number

- the numbers of the two nodes at the endpoints of the element 


\section{$-29-130$}

- the transmissivity of the element

- the length of the element

- the identification number for the fracture on which the element lies (this is printed only for reference and not used by the next program). 


\subsection{PROGRAM RENUM}

The program RENUM was designed to better use the memory space and reduce tha computing time necessary in the simulation process of the hydraulic behavior of fractured rock. It simplifies fracture networks for a more efficient computation. The code merges the nodes which are 100 close to each other, removes all dead-end clusters which do not conduct fluid and renumbers the mesh in an efficient way. RENUM is a part of a chain of programs developed at LBL, and can treat the uutput of FMG or CHANGE (Billaux and Long, 1988).

In two dimensions (Figure 3.1), fractures are assumed to be line segments (Long, 1983), and are generated by FMG as explained in Chapter 2. The chain of programs oullined in Figure 1.1 also includes three-dimensional codes. FMG3D generates random disc (Gilmour et al, 1986) networks. The discs can be used by program CHANGE (Billaux and Long, 1988) as the supports on which random channels are generated (Figure 3.2). The channels generated on the discs constitute a three-dimensional network of line elements. The problem of computing Now and transport in such networks is numerically exactly the same as computing the flow in two-dimensional line-segment networks (however the number of nodes and elements involved may vary considerably). RENUM, initially built to process two-dimensional networks, can therefore also handle three-dimensional channel networks generated by CHANGE.

In steady state, the flow problem is fully defined after the conditions on the boundaries have been given. We define the nodes as the intersections between line elements or between a line element and a boundary. Solving for the flow in each line element and the potential head at each node amounts to solving a system of linear equations, the number of unknowns being the number of nodes. 

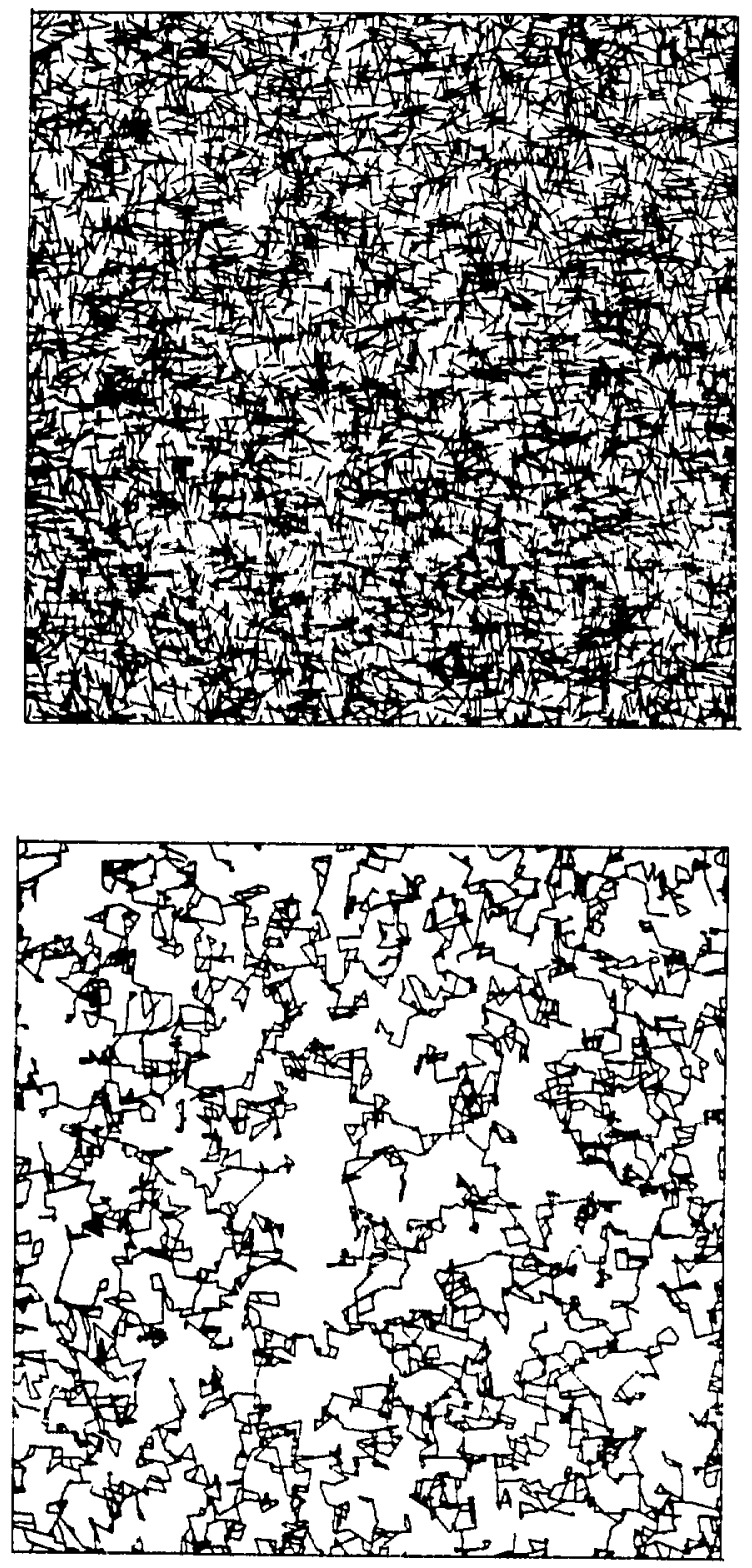

XBL $881-247$

Figure 3.1. Two-dimensional fracture mesh. (a) fractures generated pseudo-randomly, (b) same mesh with simple dead-ends removed. 

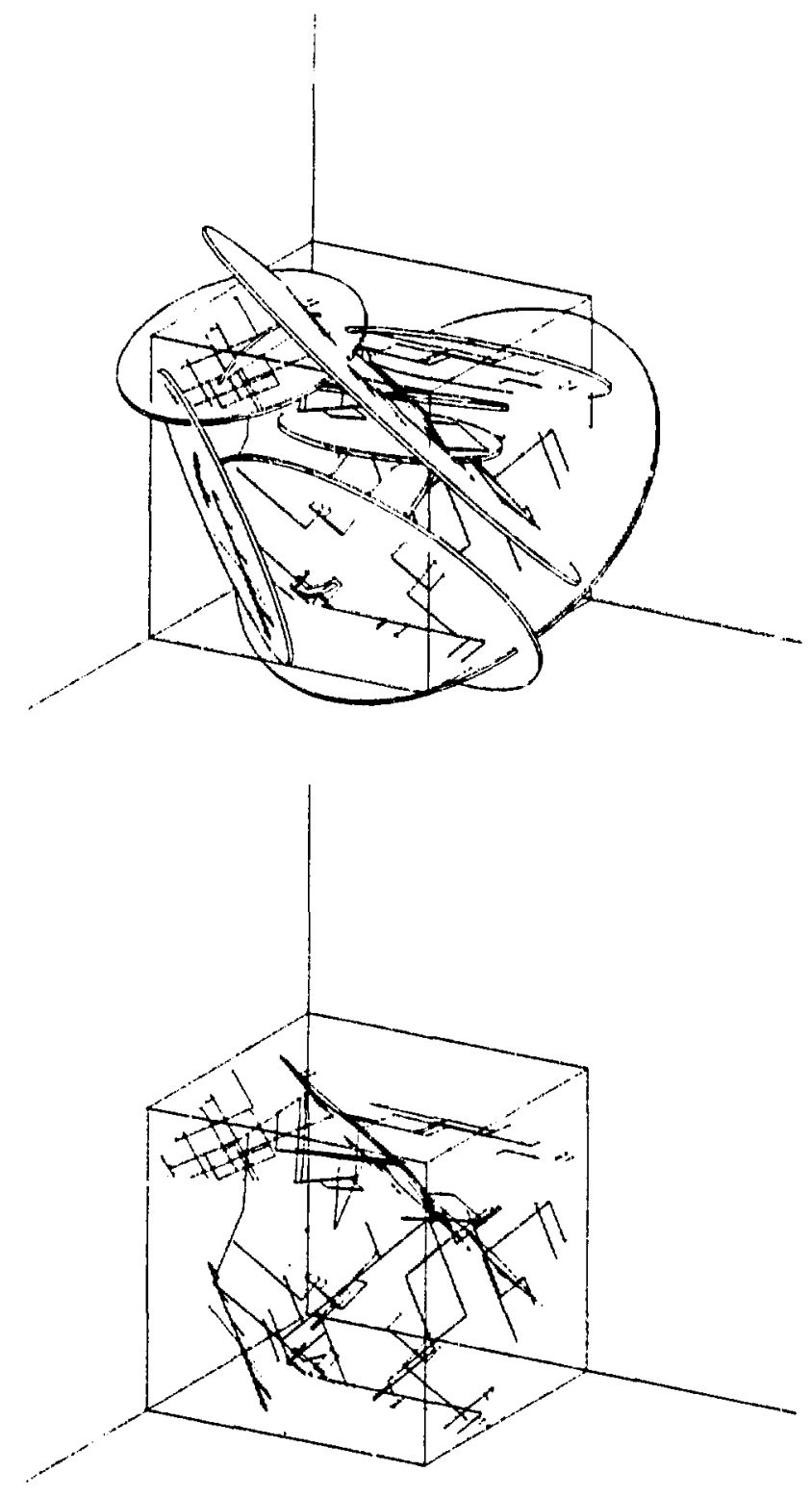

XBL $881-249$

Figure 3.2. Three-dimensional channelized fracture mesh. (a) random discs and channels, (b) channels only. 


\subsection{Merging Endpoints of Short Elements}

Due to the fact that large differences between the lengths of the elements can leati to an ill posed problem (the matrix to be solved is not positive definite within the precision of the computer), very shor elements are discanded and their ends are merged. This is done in submutine MERGE.

In the discarding process we use the thrie arrays inode( $2, i)$, intu(i) snd ibs $(2, i)$. The program loops over the elements and compares their endpoints. If the coordinates of these two nodes are equal then one of them is discarded as follows:

$i$. If the two nodes $i$ and $j$ that form the element are both boundary nodes, node $i$ is discarded by selting inew $(I)=j$. In this way, any later reference to node $\mathrm{I}$ will be pointed instead to node $\mathrm{j}$.

2. If one node $i$ is a boundary node and the other $j$ is not, node $j$ is discarded by setting inew(j) $=i$.

3. If both nodes $i$ and $j$ are interior, then node $j$ is discarded (inew $(j)=i$ ).

In this way, no connection to the boundaries is cut.

When an inner boundary condition with imposed flux is specified, all the nodes on Litis boundary are merged. In this way, the inner hole is modelled as an infinite permeability medium.

\subsection{Discarding Dead-ends}

Some definitions from graph theory are useful in descriting the algorithm used to discard dead-ends (Billaux and Fuller, 1988). A connected graph is one in which any two nodes are connected to each othe: by at least one path. In our case, this path tan be through fractures or boundary lines or both. By extension, a biconnecied graph is one in which any two nodes are connected to each other by at lezst two tctally distinct paths. In a given graph, a biconnected component is a maximum biconnecied sub-graph, i.e., a part of the graph as big as possible while still being biconnected. It is connected to the rest of the graph by only one node, which 
is then called an articulation point.

We want to keep any node which is connected to the boundaries by at lcast two distinct paths as this node will be on a connected flow path. Selecting the "active mesh" then amounts to selecting all biconnected components containing at least two boundary points. The algorithm used in subroutine PATHS identifies and lumps together all the biconnecied components including two boundary nodes, and does not attempt to distinguish between the other ones, thus saving some time. To achieve this, breadth-finst tree search is performed starting from all boundary nodes as initial points, as explained below. Figure 3.3 shows a simple mesh used to illustrate the description of the algorithm.

The breadth-fins search is a simple way of examining once every node connected to the initial points. It is performed iteratively by scanning the nodes in successive levels defined as follows. Level one contains the initial nodes. Level two contains all nodes connected to ievel one by a line element. Level $\mathbf{n}+\mathbf{1}$ contains all the nodes connected to a node in level $\mathbf{n}$ by $a$ line element and which are not already in level $\mathbf{n}$ or $\mathbf{n}-1$. Note that node connected to a node in level $n$ by a line element cannot be in a level lower than $n-1$. Otherwise the node in level $\mathbf{n}$ would have to be in a Jower level.

The process is initialized by putting in level one all the initial nodes. Then a loop over the levels is started. For any level, each node in the level is studied to find all the nodes connected to it. If a new node is encountered, it is put in the next level. At that time, we call the node we are examining the "study rode." Once all the nodes of given level have been screened, the loop over the levels is incremented and screening of the nodes in the next level begins. This goes on until at the end of the screening of a level the next kevel is cmpty. During the scarch, the nedes are numbered sequentially as they are encountered. In this process we do not encounter any nodes which are isolated from the boundary, therefore, these nodes are automatically not included in the cataiogue.

The boundary nodes are the initial points or "sources" for the first search. A sufficient condition for any node to be active is to be reached by two branches of the search originated 


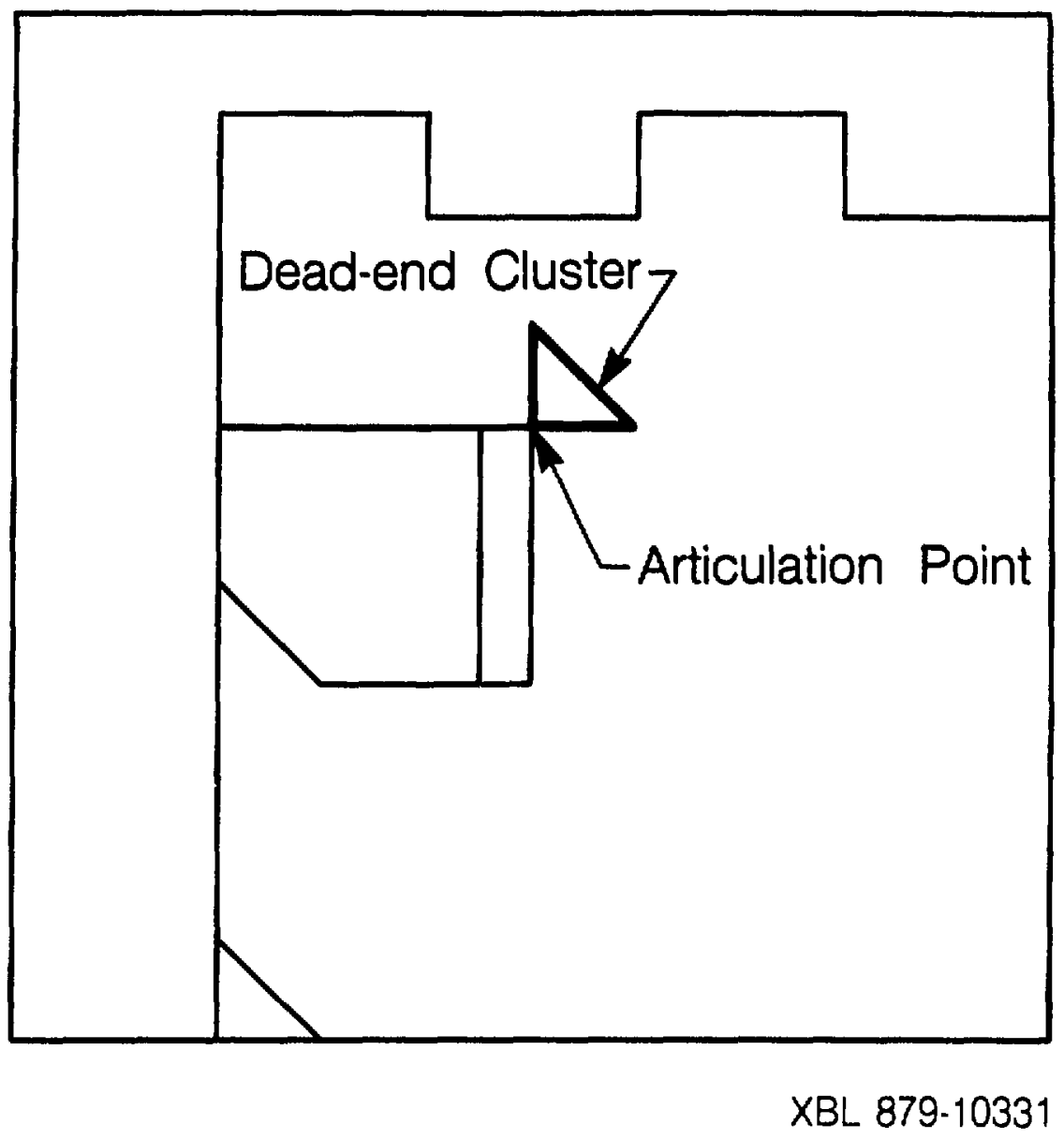

Figure 3.3. Si.nple mesh with dead-end highlighted. 
from different sources. For any node we encounter, we remember on which branch it is by recording the source from which this branch originated. Therefore, the following rules are followed when studying a given node during the scarch and looking for all nodes connected to it by a line clement:

- If a new node is encountered, we record the number of its source. This number is in fact the source number of the current study node from which we have just found the new node. One exception is the case when the study node is a source node. In this casc the source of the new node will be the study node itself. The new node is also added to the list of nodes in next level.

- If the node we encounter has bren found carlier in the search, it already has a source number. We compare this source number with the number ns we would like to assign to it if the node was new. If they are identical, both the study node and the node we have encountered are part of the same branch of the search tree and the scarch goes on. On the contrary, if the two sources are different then we have found two active nodes.

An active node is flagged by setting its source to zero. When this node is studied later in the search, it will be considered as a source itself, so that its number will be the source number of any new node we will find connected to it. To enable an active node to act as a source, we need to find that it is active before it becomes the study node. Since we study the nodes in the order in which we renumber them, this will be the case if its number is higher than the number of the current study node. Once this is done, the scarch resumes.

Once the breadth-first search is terminated, we have completely renumbered the part of the mesh connected to the boundaries (Figure 3.4). The numbering is such at this stage that when considering any two nodes, the node with the lower number is either closer or as close to the boundaries as the node with the higher number. By closer, we mean that we need to pass through less line elements to go from the boundaries to the node. We can now derive a second sufficient condition for a node to be active. Consider a dead-end cluster (Figure 3.5). 


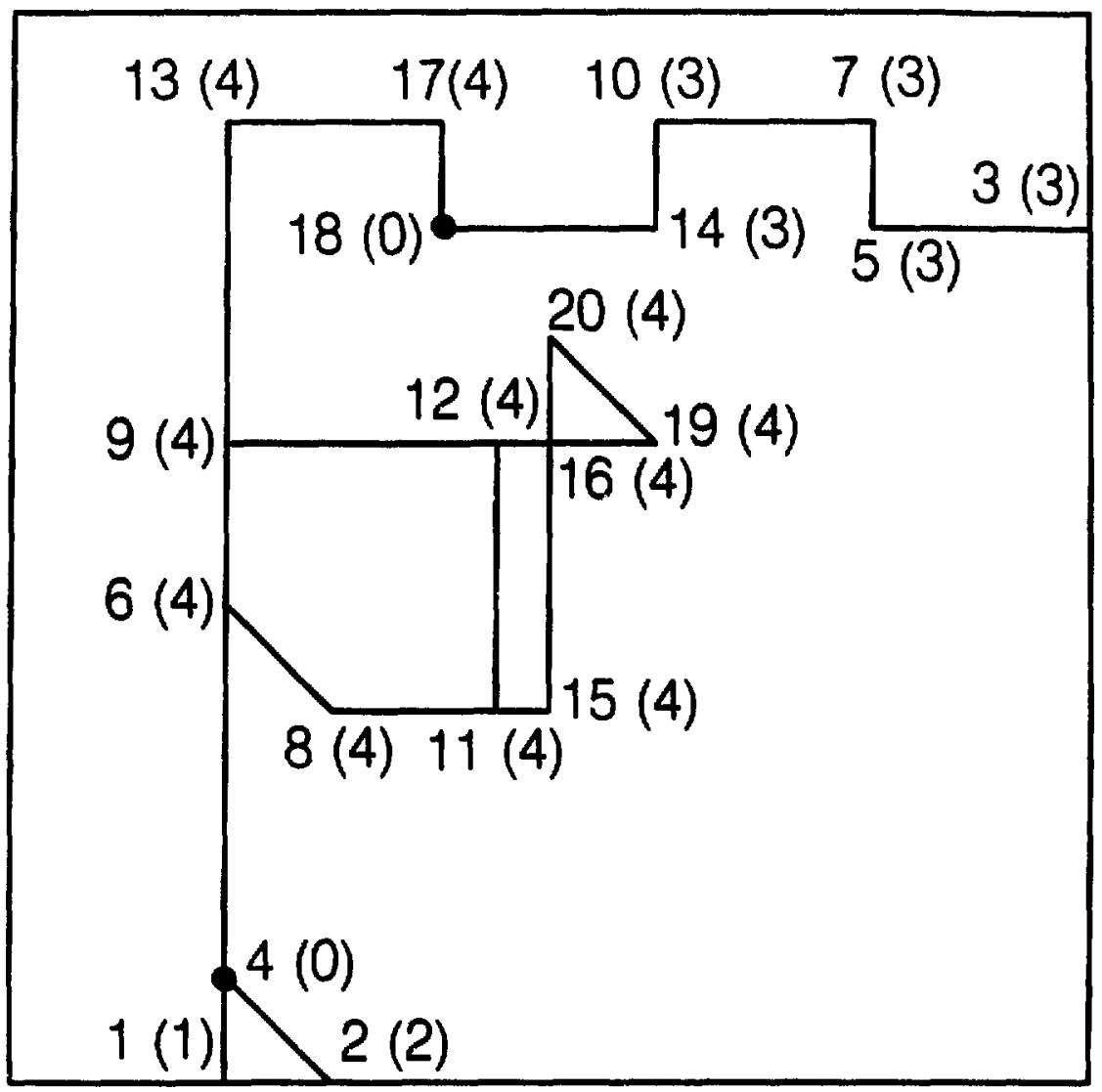

- Node Flagged as Active

9 Node Number

(2) Source Number

XBL $879-10330$

Figure 3.4. Mesh from Figure 3.3 at the end of the first search. 


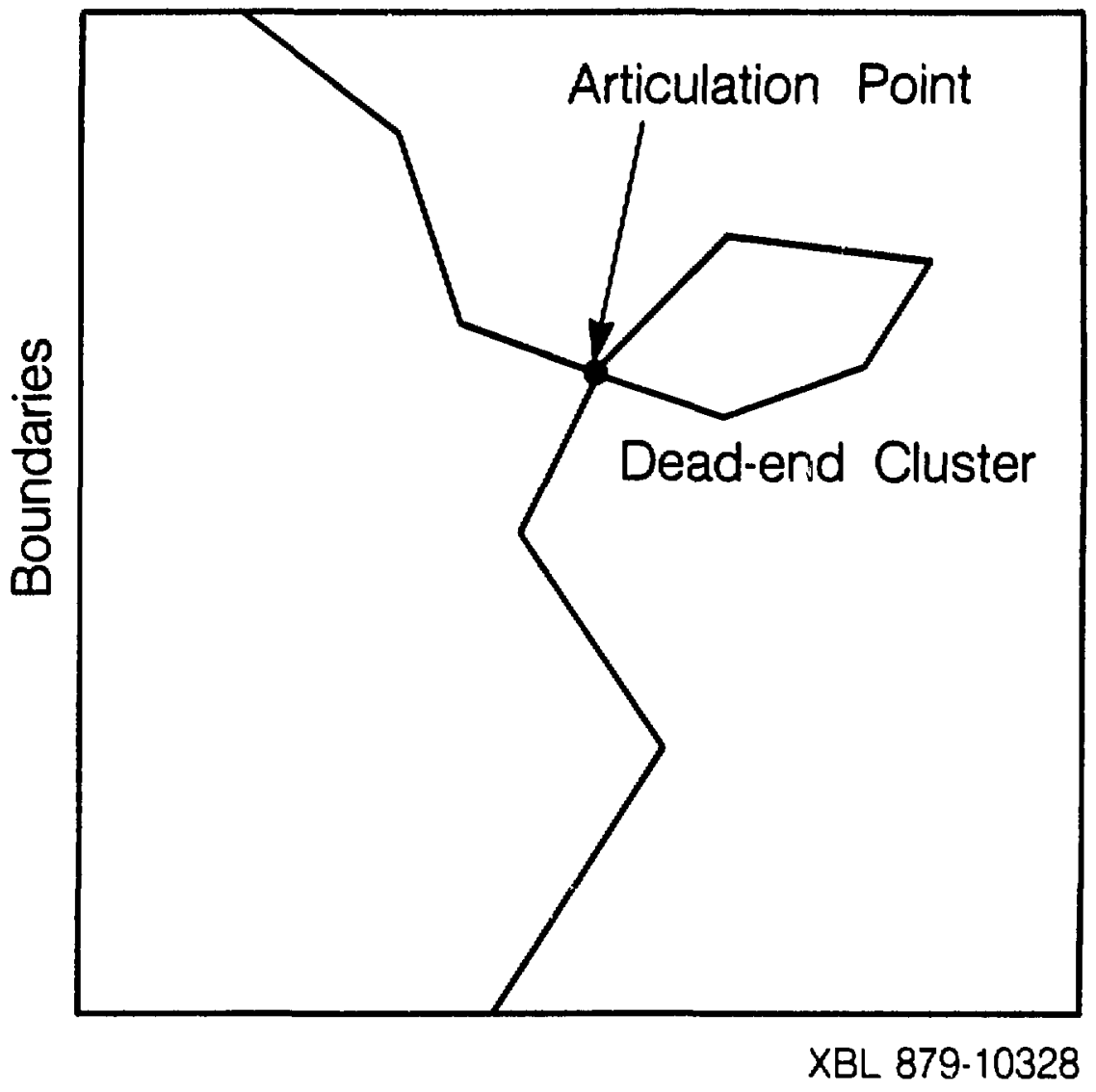

Figure 3.5. Second sufficient condition. Respective positions of boundaries, articulation points and dead-end cluster. 
We must pass through its articulation point to reach the dead-end from the boundary. Therefore the nodes in the dead-end have higher numbers than the articulation point. Reversing this proposition, we find that when we jump from one node to another along a line element, if the node number decreases, we cannot be entering a dead-end cluster. The second sufficient condition is therefore, that a node is active if it is connected by a single line element to an active node with a number higher than itself.

We use the above sufficient condition to flag active paths, that is paths in which all the nodes are active. Starting in sequence from each of the nodes flagged as active during the previous search, a restricted breadth-first search is performed. When studying a given node, instead of recording all the nodes connected to it by a line element, we select only those with a lower number. Because we started from an aclive node, all the nodes we go through are active and we flag them as such.

We may not be flagging all the active nodes in the mesh, since the condition we are using is sufficient but not necessary. For example, in Figure 3.4, nodes 8, 11, 12, 15, 16 will not be flagged. We thus will need to study again the part of the mesh which has not been flagged as active yet. To initialize this next forward search we will need to know where there are branches we did not select in the restricted search. So during this downward search, we make as initial node for the next search any node we encountered that is connected to a node not flagged active and with a higher number. Figure 3.6 shows the example mesh at the end of the first downwand search. Node 9 qualified to be an initial point for the next forward search because it is connected to mode 12 . Node 6 qualified also because it is connected to Node 8 .

Starting from the source nodes defined during the downward search, we perform the same forward search as before, but avoiding all nodes already known to be active. We renumber the nodes we encounter in sequence, assign to them new sources, and flag mone active nodes in the same manner as before. The dowliward search is then performed starting from the newly found active nodes only, and the process is repeated until all active nodes have been flagged (Figure 3.7). 


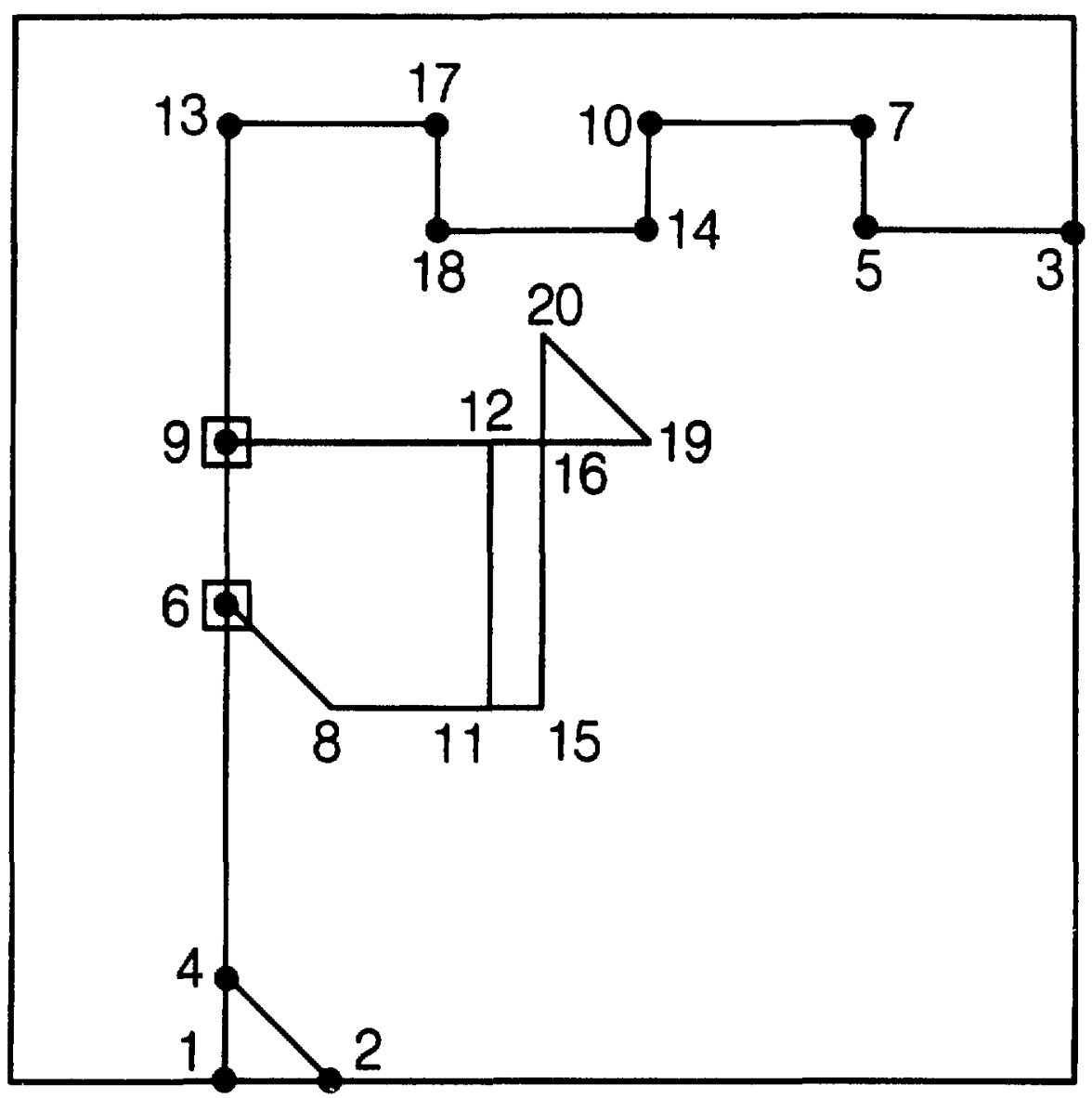

- Node Flagged as Active

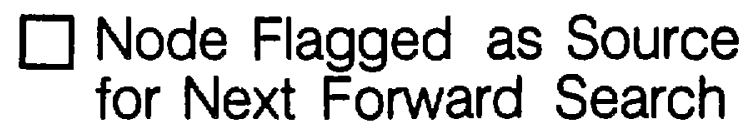

$$
\text { XBL 879-10333 }
$$

Figure 3.6. Mesh from Figure 3.3 at the end of the first downward search. 


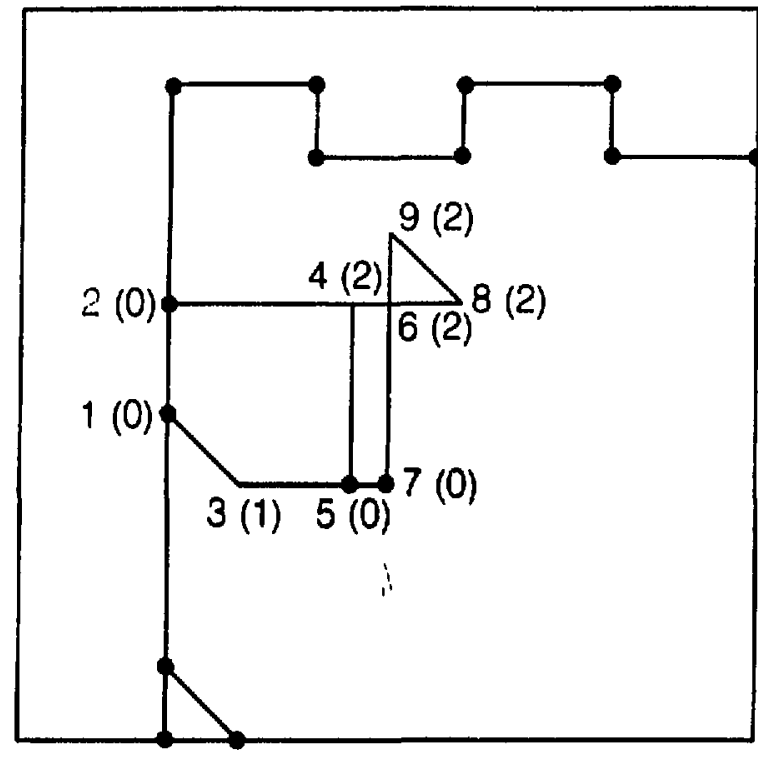

- Node Flagged as Active

9 Node Number

(2) Source Number

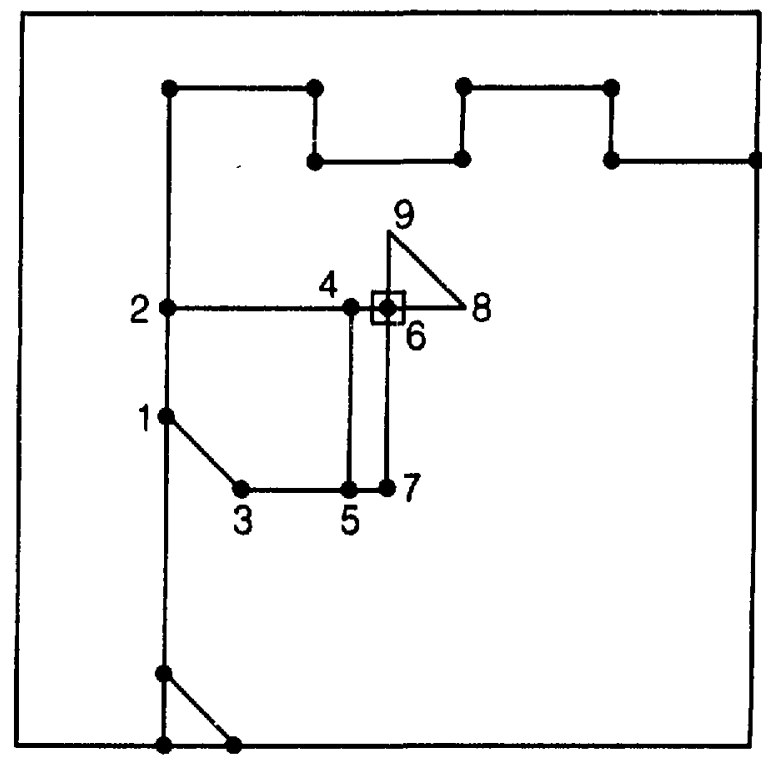

- Node Flagged as Active Node Flagged as Source for Next Forwarn Search

Figure 3.7. Mesh from Figure 3.3, second loop of searches. (a) forward search, (b) downward search: only one source is left for the next forward search. The algorithm stops, nodes 8 and 9 are discarded. 
The process is stopped after a downward search if not more than one new source node has been flagged. After a forward search it is stopped if no new active node has been flagged.

\subsection{Node Renumbering and Output}

\subsubsection{Banded Matrices}

The bandwidth of a matrix is the maximum of the difrere. lee hetween the numbers of two nodes in the same row, plus one. For a symmetric matrix, only half of the matrix is generilly stored, either the upper or the lower triangle bounded by the diagonal. So the hall-bandwidth is detined for a symmetric matrix as the maximum distance along any row between the diagonal and a non zero off diagonal tem, plus one. Numerical algorithms for the solution of linear systems take advantage of a narrow bandwidth in various ways. The most common way is to store only a number of terms equal to the bandwidth for each row of the matrix, thus reducing the storage requirements and the number of operations needed. If either a complete or an incomplete Choleski decomposition is performed, only terms within the band are filled in (i.c., are changed from zero to non zero). Thus the narrow bandwidth also drastically reduces the storage and computer time requirements. These solving procedures aie discussed in more detail in paragraph 4.2 .

\subsubsection{The Cuthill-McKee Algorithm}

The algorithm used to minimize the bandwidth was published by Cuthill and McKec (1969). It consists of searching the network level by level as explained in Section 3.2 and renumbering the nodes as they are reacled. For example, in Figure $3.8 \mathrm{a}$ if node one is put in level one, then level two consists of nodes (2 and 8), level 3 of nodes ( 3 and 6), and so on, and the nenumbering yields the node numbering shown in Figure 3.8b. The matrix obtained has a half bandwidth of 4 as compared to the matrix in Figure 3.8a which has a half bandwidth of 8 .

The search is initialized by putting all boundary nodes on side one in level one. Once all nodes connected to side one have been screened, all boundary nodes on side two which have 

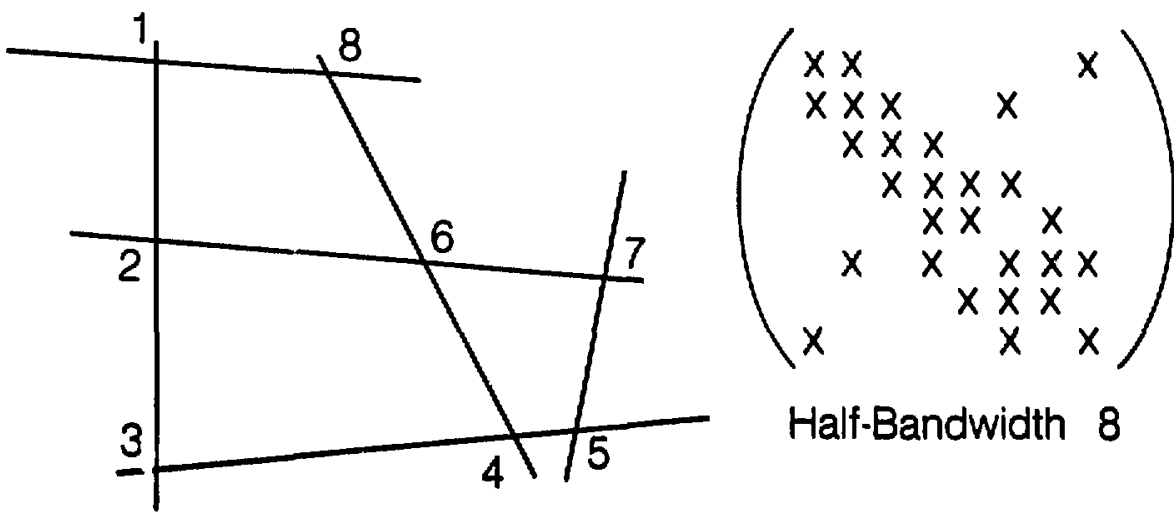

Half-Bandwidth 8

a) Old Numbering and Matrix
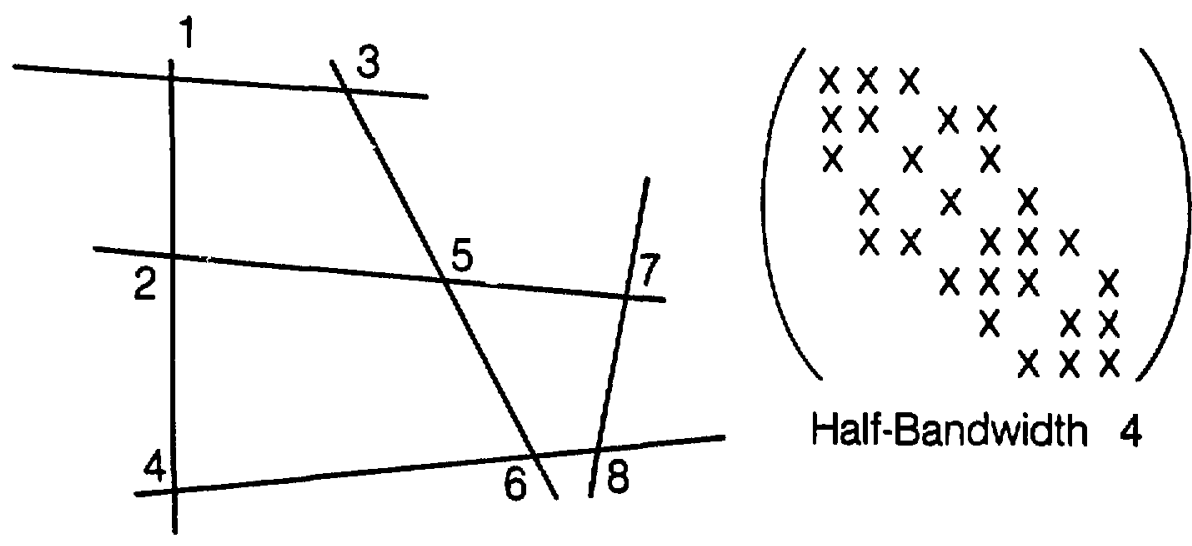

Half-Bandwidth 4

b) New Numbering and Matrix

Figure 3.8. Node renumbering, after Robinson (1982). 
not yet been sereened are put in thi next level and the search is reinitialized. This goes on until all sides have been exhausted (four sides in 2 dimensions or 6 sides in 3 dimensions).

Subroutine PLOT then writes plotting files, linesnn.dat, with $\mathrm{nn}=01,02,03$ etc., for each flow region studied. Then, the bandwidth is compuled by PROUT, and this subroutine prints the finite element network for use by programs LINEL (Chapter 4) or 'TRINET (Karasaki, 1987). 


\subsection{PROGRAM LINEL}

LINEL computes the steady state flux through a mesh of line elements previously processed by program RENUM. LINEL, was first developed by Wilson (1970).

For a given set of boundary conditions, the head at each node and the llux in each clement are computed. The output of the program consists of the sum of the fluxes through each side of the flow region.

The program can deal with two dimetisional meshes generated by FMG or with three dimensional meshes generated by FMG3D-CHANGE (Gilmour et al., 1986; Billaux and Long, 1988). For two dimensional problems, study regions can be defined as an option. These are smaller rectangular regions centered in the flow region. Whenever such regions are specified, the flux through their boundaries and the average gradient inside them are also computed.

\subsection{Building the Linear System of Equations}

Using Darcy's law for each clement and writing the conservation of mass at each node of the network yield the system of linear equations that needs to be solved given the boundary conditions.

\subsubsection{Governing Equations}

In each line element, Darcy's law states that the flux through the element is proportional to the gradient of charge in the element, the proportionalily constant being the transmissivity of the element. So given an element of length $l_{i j}$ and transmissivily $t_{i j}$ joining to nodes $i$ and $j$, and given the charges $h_{i}$ and $h_{j}$ at nodes $i$ and $j$, the flux $Q_{i j}$ from $i$ to $j$ is given by:

$$
Q_{i j}=t_{i j} \frac{\left(h_{i}-h_{j}\right)}{1_{i j}}
$$

At each intemal node there is no creation of mass, except if the node is specified as a source. 
So for all i's, we have

$$
\sum_{j} Q_{j i}=q_{i}
$$

where $q_{i}$ is the imposed flux at node $i$, which is zero for most or all intemal nodes. The sum is over all nodes $\mathbf{j}$ connected to node $\mathrm{i}$ by one line element. Nole that this equation cannot be written for imposed-head nodes, where some mass is effectively entering or leaving the mesh. At these nodes, we simply write the identlty between the head and the imposed head.

\subsubsection{Linear System}

The linear sysem is ot tained by simply writing Equation 4-2 for all nodes, with the $Q_{i j}$ s written in terms of the $k_{i}^{\prime} s$ using Equation 4-1. If the mesh has n nodes, the matrix will then be of onder $n$.

Each line $i$ of the linear system has the form:

$$
\sum_{j} \frac{t_{i j}}{l_{i j}} h_{i}-\sum_{j} \frac{t_{i j}}{l_{i j}} h_{j}=q_{i}
$$

where the summation on $j$ 's is the same as in Equation 4.2 .

If this system of equations is written:

$$
A \mathbf{h}=\mathbf{b}
$$

then from Equation 4.3, the diagonal terms in $A$ are

$$
A_{i j}=\sum_{j} \frac{t_{j j}}{l_{i j}}
$$

the off diagonal terms are:

$$
A_{i j}=-\frac{t_{i j}}{l_{i j}}
$$

if an element ij exists; otherwise,

$$
\mathbf{A}_{\mathrm{ij}}=0
$$

and the $b$ tems are:

$$
b_{i}=q_{i}
$$


The above terms are complete if there are no imposed head boundary nodes. If a node $k$ has iis head imposed, the line $\mathbf{k}$ of the system reduces to

$$
\left[\sum_{j} \frac{t_{k j}}{l_{k j}}\right] h_{k}=\left[\sum_{j} \frac{t_{k j}}{l_{k j}}\right] H_{k}
$$

where $H_{k}$ is the imposed head.

We can then rewrite Equation 4-3 separating the nodes into non-imposed head nodes and imposed head nodes:

$$
\sum_{j} \frac{t_{i j}}{l_{i j}} i_{i j}+\sum_{k}-t_{i k} h_{i}-\sum_{j} \frac{t_{i j}}{l_{i j}} h_{j}-\sum_{k} \frac{t_{i k}}{l_{i k}} H_{k}=q_{i}
$$

where node $i$ is a non-imposed head node, the sum of $j$ 's is over all non-imposed head nodes connccted to node $i$ by a line element, the sum in $k$ 's is over all imposed-head nodes connecled to node $\mathrm{i}$ by a line clement. Passing the fourth term in the left hand side (knnwn term) to the right, we obtain:

$$
\sum_{j} \frac{t_{i j}}{l_{i j}} h_{i}+\sum_{k} \frac{t_{i k}}{l_{i k}} h_{i}-\sum_{j} \frac{t_{j j}}{l_{i j}} h_{j}=q_{i}+\sum_{k} \frac{t_{i k}}{l_{i k}} H_{k}
$$

The left hand side represents matrix $A$, and the right hand side is the $b$ vector. So we obtain the final expression for the terms of $A$ and $b$ :

- diagonal terms in A:

$$
A_{i i}=\sum_{j} \frac{t_{i j}}{l_{i j}}
$$

where the sum in $j$ 's is over all nodes $j$, either imposed head or not, connected to node $\mathbf{j}$ by a line element

- off diagonal terms in A:

if an element $\mathrm{ij}$ exists and neither node $\mathrm{i}$ nor node $\mathrm{j}$ is an imposed-head node, then

$$
A_{i j}=-\frac{t_{i j}}{!_{i j}}
$$

otherwise

$$
A_{i j}=0
$$


- terms in b:

if node $i$ is a non-imposed head node

$$
b_{i}=a_{i}+\sum_{k} \frac{t_{i k}}{l_{i k}}
$$

where the sum in k's is over all imposed-head nodes connected to node $i$ by a line element.

If node $k$ is an imposed head node,

$$
b_{k}=\sum \frac{t_{j}}{L_{k j}}
$$

where the sum in $j$ 's is 'vver all nodes connected to node $k$ by line clement.

\subsubsection{Implementation}

Subroutine CPHI builds $A$ and $b . A$ bop is performed over all the line elements in the network. For each of them, the numbers of the nodes $x$ the extremities are retrieved, the right terms in the dingonal of $A$ are added, then depending on the status of the two nodes (either imposed head or not), the proper terms are added into $b$ or put of the diagonal into $A$. Note that several (up to three) b vectors can be built at the same time, to compute, for example, flow in a given mesh under gradients in several different directions.

In order to save computing time when solving the linear system, when filling matrix A and vector $b$, the lines of $A$ and $b$ corresponding 10 imposed-head nodes are switched to the bottom of the tables. Then the onder of the matrix passed to the linear solver will be onty the number of nodes where head is not imposed. In this way, the program avoids solving the lines of the system where it reduces to an identity.

For two-dimensional networks, the default number of boundary conditions is two. The boundary conditions used are: 1) the conditions imposed by the users, and 2) the same conditions rotated $90^{\circ}$ (Figure 4.1). In this way, two different directional permeabilities are found at the same time in the process of computing a porous medium equivalent tensor (see Chapter 5 ). This default setup is overridden whenever an imposed flux boundary node is present in the 

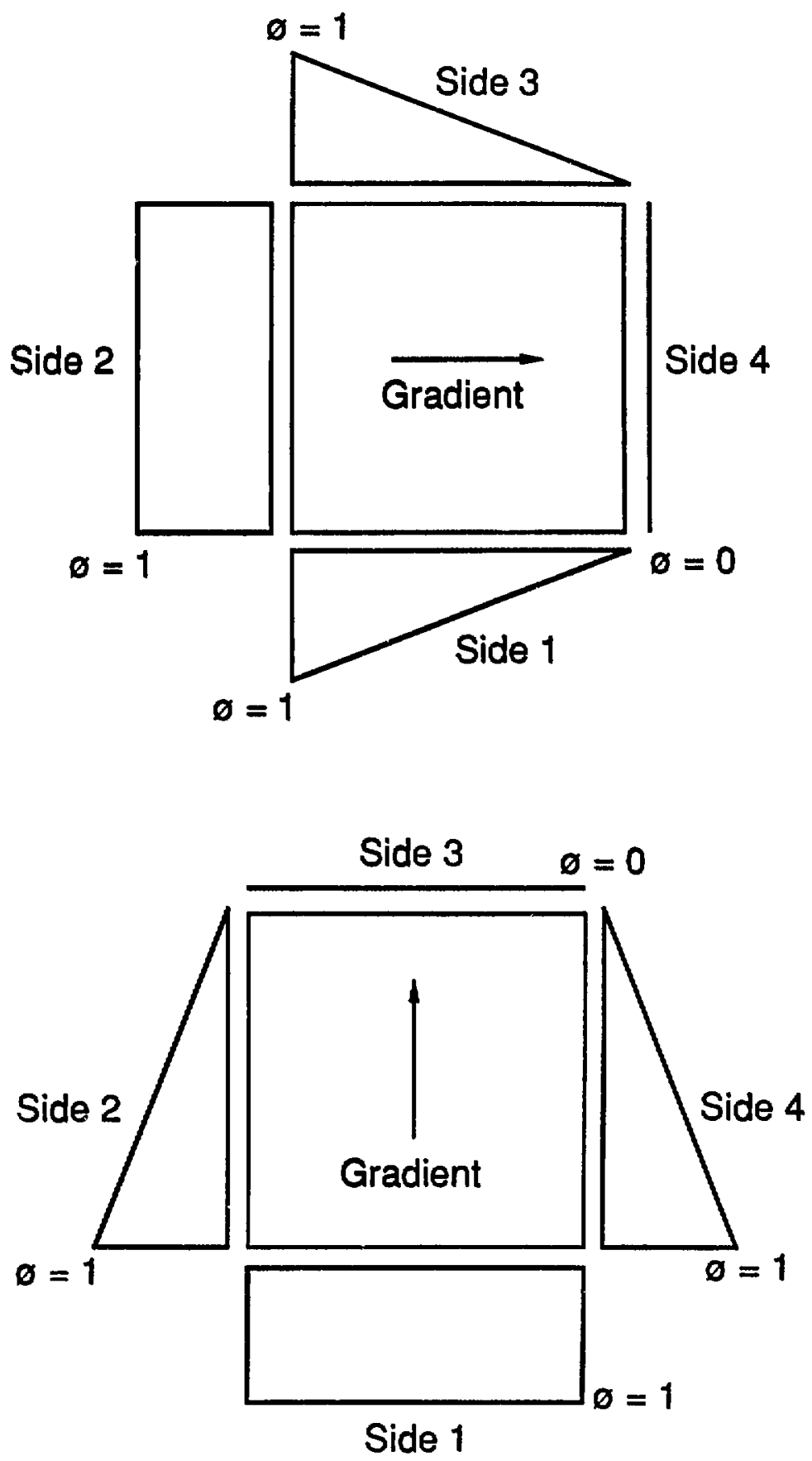

XBL 882-10062

Figure 4.1. Two sets of boundary conditions for directional permeability. 
mesh.

For three-dimensional networks, the number of differing sets of boundary conditions is input by the user, and each set of boundary conditions is fully defined by the user.

The way matrix $A$ is stored in the computer depends on the solver dsed later. The solver requires $A$ to be stored in banded fashion.

\subsection{Solving the Linear System}

A system of equations representing flow in a line element network has scveral interesting properties. The matrix $A$ is symmetric positive definite, and is also generally sparse with a narrow bandwidth. The symmelry follows direclly from the expression for the off-diagonal terms in $A$. The fact that $A$ is both positive and definite stems from the relationship between the off-diagonal and diagonal terms in every line. From the construction of A (Equations 4-6, 4-7 and 4-8), it follows that:

$$
A_{i j}<0
$$

for all $i$ and for all $j$ such that element $i j$ exists.

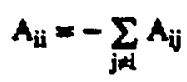

if no node $j$ is an imposed head node;

$$
A_{i j}>-\sum_{j i j} a_{i j}
$$

if at least one node $\mathbf{j}$ is an imposed-head node.

Simple algebra then can prove a sufficient condition for positive definiteness which is that for any vector $x$,

$$
x^{\prime} A x>0 \text {. }
$$

Recall that an off-diagonal element of matrix $A, A_{i j}$ is non-zero only if a line element joins the two nodes $i$ and $j$. This implies that on a given line $i$ of $A$, there will be as many off-diagonal non zeros as there are line elements having node $i$ as an endpoint. Experience shows that almost all nodes are the endpoints of two to four line elements. A node can be the endpoint of 
more than four elements in two instances only. 1) If three fractures intersect at the same point or at almost the same point, program RENUM merges the several resulting nodes into one. 2) All the nodes inlersecting a given imposed flux hole (i.e., a well) are also shrunk into one by RENUM. So the number of non-zeros on any given line of $A$ averages less than five (four off-diagonals plus one diagonal). Considering that networks of 10,000 nodes are not uncommon, the matrix A for such networks is made of $99.95 \%$ zeros and $0.05 \%$ non zeros. In fact, the number of non-zero off-diagonals we need to store can be cut in half because of the symmetry.

The narrow bandwidth of the system is discussed in Chapter 2. Note that even for a sparse system, renumbering the nodes to minimize the bandwidth is efficient and reduces overall computer time.

The solver SYMSOL is a fortran subroutine that solves the linear system of equations with A stored in lower triangular banded form. SYMSOL uses the lower triangle decomposition method to solve the system of equations. This solver is implemented in FORTRAN-77, so it is easily portable to any computer.

\subsection{Computing Fluxes}

The solution of the linear system is an array containing the hydraulic head at each node in the network. The flux entering the system at each imposed head boundary node is computed. The sum of the fluxes through each side of the flow region is then computed and printed. If required, the head and flux at the boundaries of a smaller study region are also computed and printed.

\subsubsection{Flow Region}

Once the head at each node is known, it is a simple matter to compute the flux through any element of the network, by using Equation 4-1. Subroutine CUNK loops over all the elements. When an endpoint i of an element is found to be an imposed-head node, the flux leaving node $i$ through the element is computed. 
Subroutine PINFO then prints the header for the output file, and optionally all the node and element characteristics together with heads at nodes and velocities in elements.

The fluxes leaving all the nodes through each boundary side are summed up by subroutine SFLUX. These fluxes are output, as well as the inverse of their square root for twodimensional cases, to be used by program ELLFMG (Chapter 5).

Also, if flux has been imposed at any node, the head al this node is printed. The average number of fractures per unit length (2-D) or area (3-D) intersecting each side of the flow region is also computed and printed.

\subsubsection{Study Regions}

For computing equivalent porous medium permeabilities in the two-dimensional case, the boundary conditions that are imposed (see Section 2.3.3) are likely to cause a consistent overestimation of the permeability, because flow may be forced through fractures or fracture clusters that only transect the comer of the region. The importance of this effect decreases as the scale of measurement is increased. This problem is handled by using "study regions" (Figure 4.2).

A study region is centrally located inside the flow region, and a border region of width $\Delta$ is left between the study region and the boundaries of the flow region. The boundary conditions are still applied to the now region sides, but fluxes are examined at the boundaries of the study region. For a large enough $\Delta$, the border effecl is all but eliminated. But another difficulty then arises in defining the gradient $\mathbf{J}$ over the study region. The gradient over the flow region is clearly defined by the applied boundary conditions. The gradient $\mathrm{J}$, over the study region, however, can be defined in one of two ways. We can take $\mathrm{J}_{\mathrm{s}}$ equal to $\mathrm{J}$ operating on the flow region, which we call the "global gradient". Altematively we can calculate a local gradient. We do this by computing the head at the intersection between any fracture and the boundaries of the study region. By taking the difference between the avcrage heads on the outflow and inflow sides, we are able to find the gradient which would theoretically be meas- 


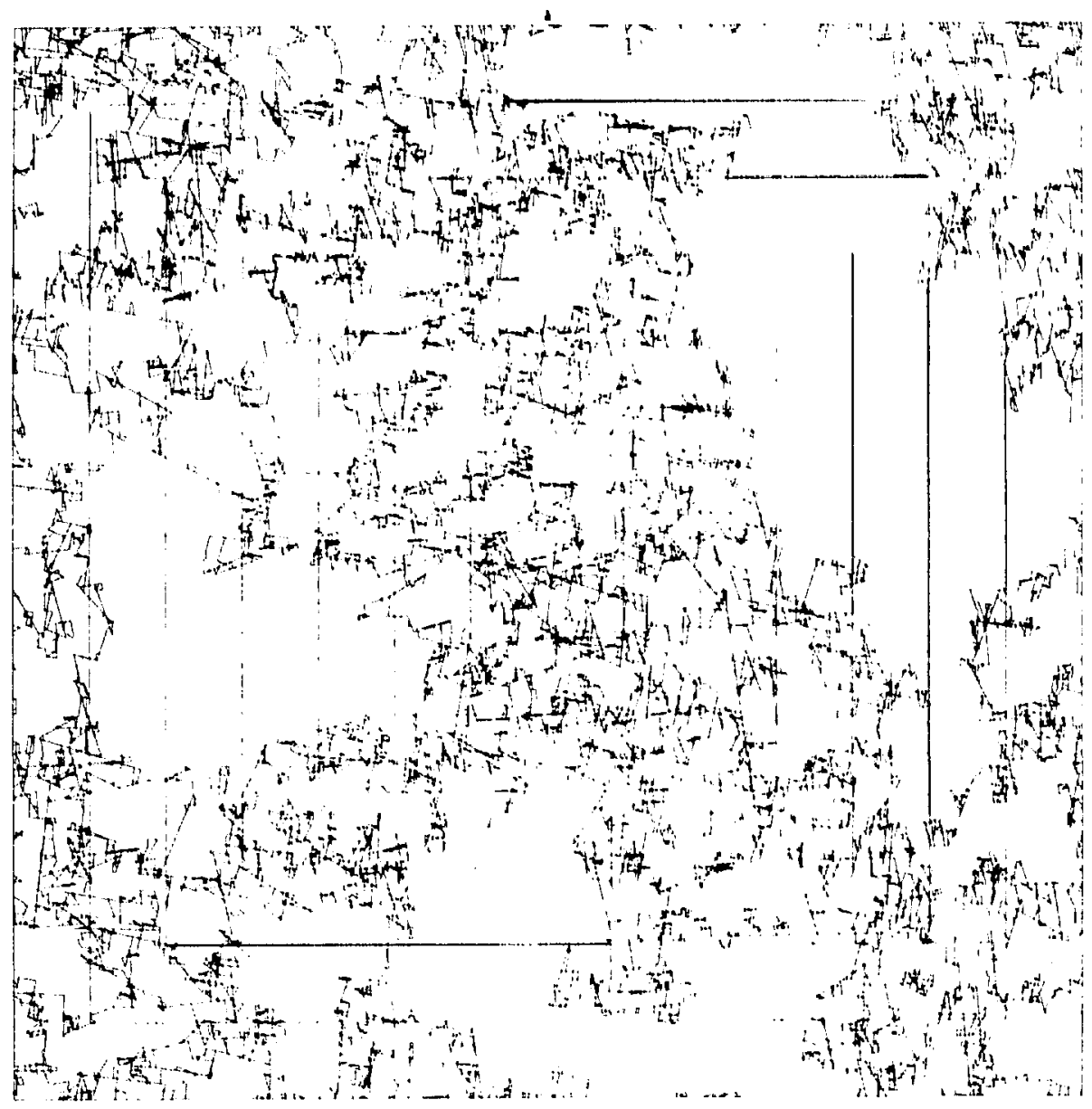

XBL 863-1170

Figure 4.2 . A $70 \mathrm{~m}$ by $70 \mathrm{~m}$ fow region with six nested study regions. 
ured in situ by monitoring hesds at the boundary of the region. Note that the global and local gradients should be identical if the medium behaves as a continuum.

Subroutine STUDY optionally performs study region computations if such regions have been specified. The intersection points between fractures and study region boundaries are determined. The head and fiux at these points are computed using the known heads at all the nodes in the network. The appropriate sums of fluxes and averages of heads are then computed, and permeabllities computed using local or global gradients are output, as chosen by the user. Nole that several (up to 20) study regions can be specified for the same run.

The intersections between each study region and the elements of the network arc found using part of the algorithm described in Section 1.2 .2 for truncating fractures lying partially outside the flow region. When an intersection is found, the flux in the element is computed using Equation 4-1. The head at the intersection is also computed using the assumption that the head varies linearly along any element. If the endpoints of the element are nodes $i$ and $j$, and the intersection is at a relative distance $t$ from node $i$ (Figure 4.3), with $t=0$ at nodis $i$ and $t=1$ at node $j$, then:

$$
h=h_{i}+t\left(h_{j}-h_{i}\right)
$$

where $h$ is the head at the intersection, $h_{i}$ is the known head at node $i$ and $h_{j}$ is the known head at node $j$. Then the head and flux are added to the sum of heads and the sum of fluxes stored for the particular side of the study region. The squares of the heads are also added up for each side in order to compute the standard deviation of heads.

Once all the elements have been screened, the average and standard deviation of heads on each side of all study regions are computed and printed. If needed the local gradient is computed, as the ratio between the difference in average heads from the inflow to the outflow side, and the size of the study region. Using the sum of the fluxes on the outflow side and inflow side, and either the global gradient or the local gradient, the average permeability is computed and printed in a file to be used as input for program ELLFMG. 
$-57-158$

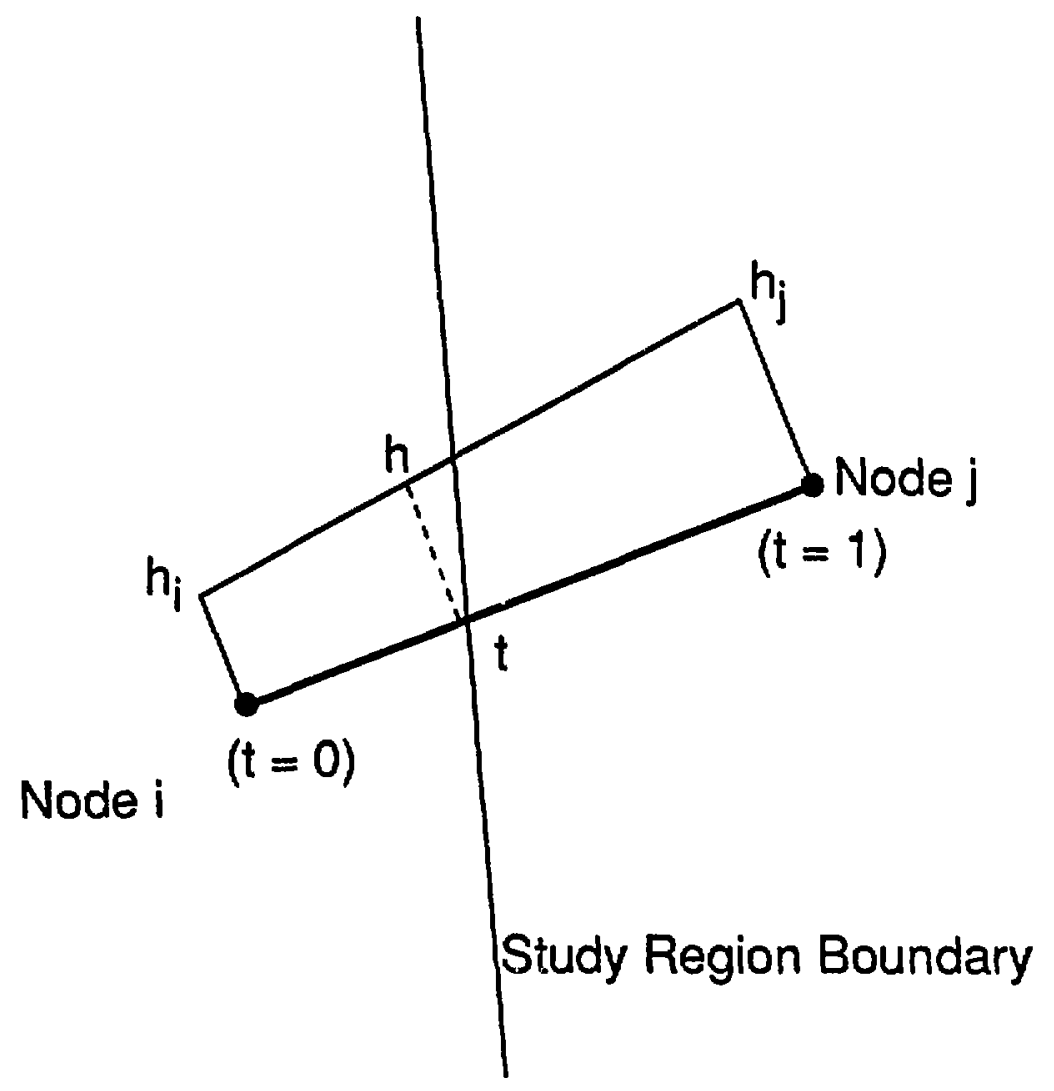

XBL 882-10063

Figure 4.3. Notations for computing the head at study region boundaries. 


\subsection{PROGRAM ELLFMG}

As mentioned before, the prognm ELLFMG is one of the links in a chain of programs, which consists of FMG, RENUM, LINEL, ELLFMG, DIMES and ELLP, built to analyze fluid flow through a two-dimensiond fracture network. The input to program ELLFMG is a list of directional permeabilities obtained by LINEL. This list is built using the ability of the programs FMG, RENUM and LINEL to process several flow regions in one run, for one given set of generated fractures. It can also be built by appending results from different runs, making sure that the same pseudo-random generation process is repeuted for each nun. An example of several flow regions for the same mesh is shown in Figure 5.1. For each of them, flow is computed under agradient from side 2 to side 4. Nove that since LINEL is able to compute flow under both a gradient from side 2 to side 4 and a gradient from side 3 to side 1 at the same time (Chapter 4), Figure $5.1 \mathrm{~g}$ (90\% rotation) corresponds to solving the same matrix as Figure 5.1a $\left(0^{\circ}\right.$ rotation). Identically, pairs of rotations $\left(15^{\circ}\right.$ and $\left.105^{\circ}\right),\left(30^{\circ}\right.$ and $\left.120^{\circ}\right)$, $\left(45^{\circ}\right.$ and $\left.135^{\circ}\right),\left(60^{\circ}\right.$ and $\left.150^{\circ}\right),\left(75^{\circ}\right.$ and $\left.165^{\circ}\right)$, each correspond to solving one linear system, From the obtained values for the directional permeability in several direction, $K_{B}(\alpha)$, ELLFMG determines the three components of the permeability tensor, $\mathrm{K}_{\mathrm{ij}}$, which fits best these results. Then the principal values (eigen values) and principal axes (eigen vectors) of the permeability tensor are computed. ELLFMG also produces a quantitative measure of the difference between the measured values, $K_{g}(\alpha)$, and the best-fit values.

\subsection{Permeability Ellipse and Permeabllity Distribution.}

For an ideal anisotropic homogeneous porous medium the directional permeability, $\mathrm{K}_{\mathbf{f}}$ measured in the direction of the gradient $(\alpha)$, is defined by the following equation:

$$
\mathbf{q}_{\mathbf{i}} \mathbf{n}_{\mathbf{i}}=\mathbf{K}_{\mathbf{z}} \mathbf{J}
$$

where $n_{i}$ is a unit vector in the direction of the gradient, $J$ is the magnitude of the gradient, and 

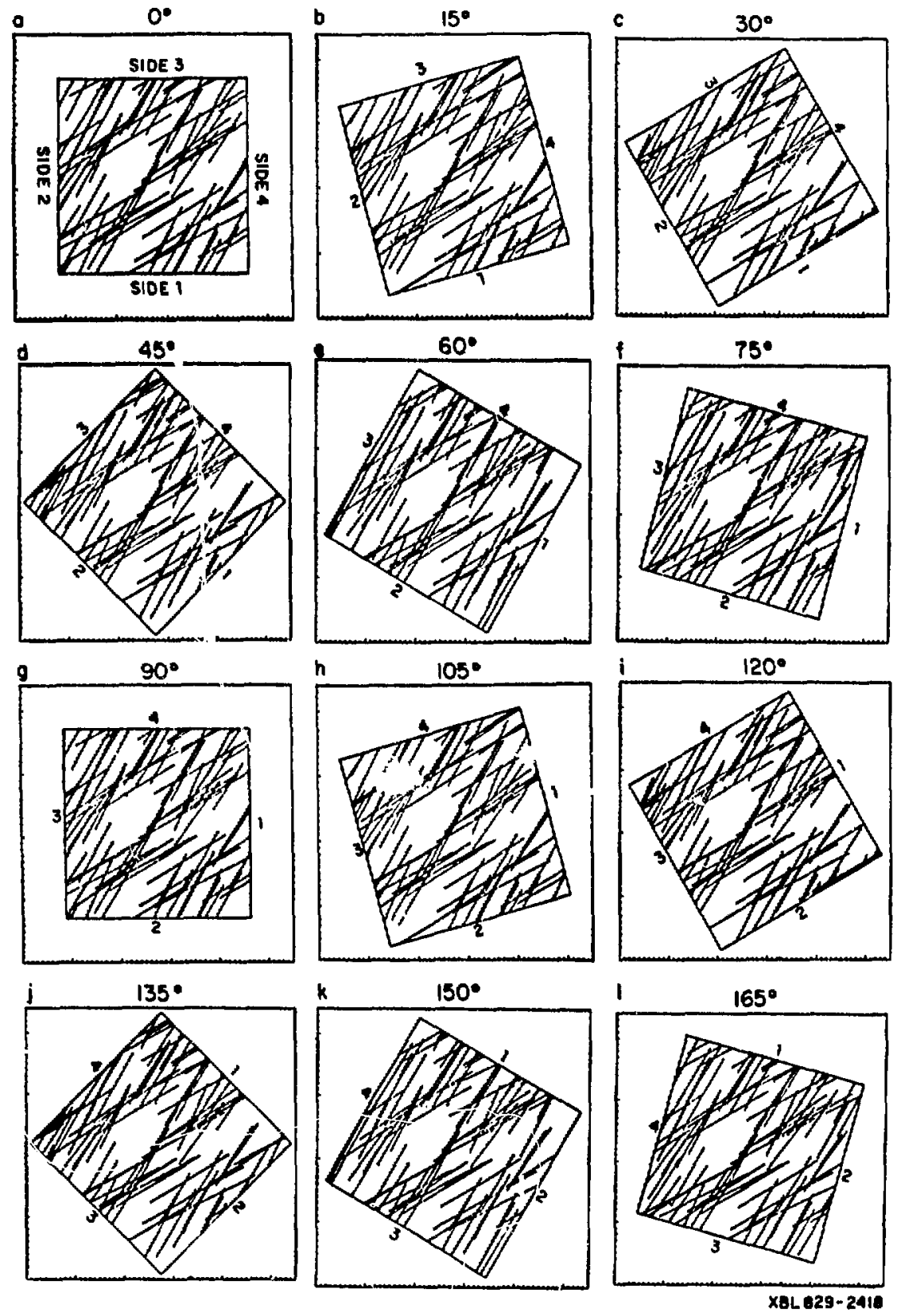

Figure 5.1. Flow regions with various orientations for directional permeability studies. 
$\mathrm{q}_{\mathrm{i}}$ is the specific flux. Solving Darcy's law for $\mathrm{q}_{\mathrm{i}}$ and substituting this into $5-1$ gives

$$
\mathbf{K}_{\mathrm{ij}} \mathbf{J}_{\mathbf{j}} \mathbf{n}_{\mathbf{i}}=\mathbf{K}_{\mathbf{8}} \mathbf{J} \text {, }
$$

and since $\mathrm{J}_{\mathrm{j}} / \mathrm{J}=\mathrm{n}_{\mathrm{j}}$ we have

$$
\mathbf{K}_{\mathbf{t}}=\mathbf{K}_{\mathrm{ij}} \mathbf{n}_{\mathbf{i}} \mathbf{n}_{\mathbf{j}}
$$

or

$$
K_{t}=K_{11} \cos ^{2} \alpha+2 K_{12} \cos \alpha \sin \alpha+K_{22} \operatorname{sil}^{2} \alpha_{1}
$$

where $n_{1}$ and $n_{2}$ are direction cosines and $n_{1}=\cos \alpha_{1} n_{2}=\sin \alpha$.

If $1 / \sqrt{K_{1}}$ is plotted in the direction $\alpha$ (the direction of the gradient), then $n_{1}=\cos \alpha=x \sqrt{K_{2}}$ and $n_{2}=\sin \alpha=y \sqrt{K_{2}}$. Equation 5.4 becomes

$$
\begin{aligned}
K_{t} & =K_{11} x^{2} K_{t}+2 K_{12} x y K_{t}+K_{22} y^{2} K_{t}, \\
1 & =K_{11} x^{2}+2 K_{12} x y+K_{22} y^{2}, \\
1 & =K_{i j} x_{i} x_{j} \quad \text { where } x_{i}=\left\{\begin{array}{l}
x \\
y
\end{array}\right\} .
\end{aligned}
$$

Equation 5-7 is the quadratic form of the equation of an ellipse, which we will call the permeability ellipse. This ellipse has semi-axes of length $U / \sqrt{\mathbf{K}_{1}}$ and $1 / \sqrt{\mathbf{K}_{2}}$ which correspond to the principal axes of the permeability tensor. Note that the major axis of the ellipse is in the direction of minimum permeability.

If each measurement of $K_{8}(\alpha)$ can be considered an independent measurement of the value of $K_{i j}$, then methods of statistics can be used to estimate the parameters $K_{11}, K_{12}$ and $K_{22}$. The statistical technique can be used on measurements of $K_{2}(\alpha)$ from different, equally incremented tirections on one fracture pattem or the combined measurements from any number of fracture pattem realizations.

\section{Distribution of $\mathrm{K}_{2}(\alpha)$}

In a random fracture paitern, the measured values of $K_{8}(\alpha)$ will not all plot in a single, unique ellipse (Figure 5.2). In order to use all of the individual measurements to derive a single, most representative set of parameters for the permeability tensor, we must assume that each measurement is independent and similarly distributed. Figure 5.2 shows an example of a 


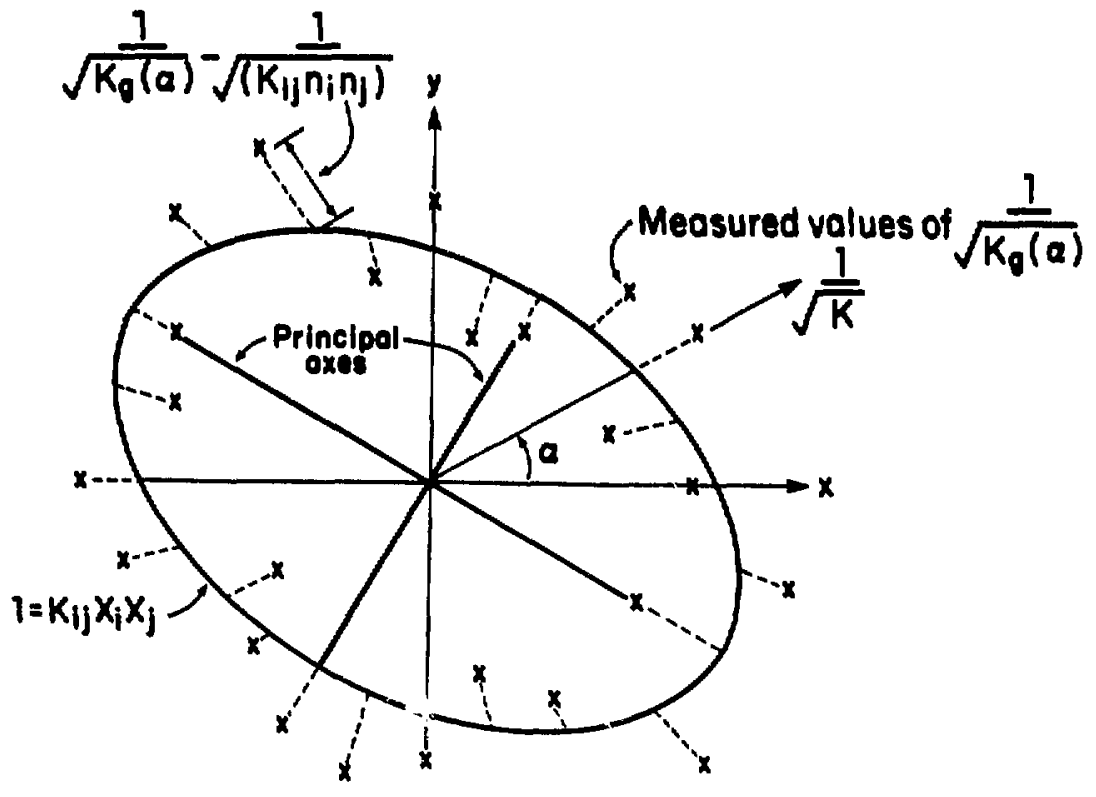

XBL $817-3316$

Figure 5.2. A set of directional permeability measurements plotted as $1 / \sqrt{K_{2}}$ in polar coordinates. 
set of measurements, $K_{8}(\alpha)$, and a possible ellipse with parameters $K_{11}, K_{12}$ and $K_{22}$. Each measurement can be assumed to be ciistributed about a different mean which is a point on the ellipse determined by $\alpha$. Therefore, the value of the mean for each measurement depends on $\alpha$. Thus, each $K_{i}(\alpha)$ is considered to be distributed with the same form but each has a different or shifted mean. The variance of each $\mathrm{K}_{\mathbf{z}}(\alpha)$ is assumed to be identical. In this way, all the measurements are considered as one population.

It would be very useful to be able to define a likely distribution function for $K_{2}(\alpha)$, bui this is not easily done. The nomal distribution does not match the data because $K_{a}(x)$ can never be less than zero. A lognormal distribution is not proper because the probability of $\mathrm{K}_{\mathrm{g}}(\alpha)=0$ is finite, not zero. Exponential, Gamma and Beta distributions also are not suitable. A normal distribution truncated at $K_{g}(\alpha)=0$ is a likely choice. Unfortunately, assuming this distribution leads to a contradiction with the basic assumption that all the measurements are members of the same distribution. At each angle $\alpha$, the mean value of the distribution is different. However, since all the distributions are truncated at zero, the difference between the mean value and the truncation limit is different for each value of $\alpha$. This means that each measurement must be a member of a different, truncated normal distribution and not just a shifted one as required in the original assumptions. Since a simple, likely, distribution form for $K_{2}(\alpha)$ which conforms to the basic assumptions cannot be identified, a least squares regression technique is used to derive estimates of the parameters $K_{11}, K_{12}$ and $K_{22}$.

\subsection{Finding the Permeability Parameters}

The regression technique used is based on a technique, discussed by Scheidegger (1954), which will also be briefly described.

In order to fird the best bit ellipse, we minimize the function $\mathbf{R}$ :

$$
R=\sum_{n=1}^{N}\left[K_{\mathrm{z}}\left(\alpha_{n}\right)-\left(K_{\mathrm{ij}} n_{i} n_{\mathrm{i}}\right)\right]^{2} .
$$

In fact we are not directly regressing to the best-fit cllipse. We are trying to find the parameters $K_{11}, K_{12}$, and $K_{22}$ which best fit the data expressed by $K_{2}\left(\alpha_{n}\right)$ in Eq. 5-5. Figure 5.3 


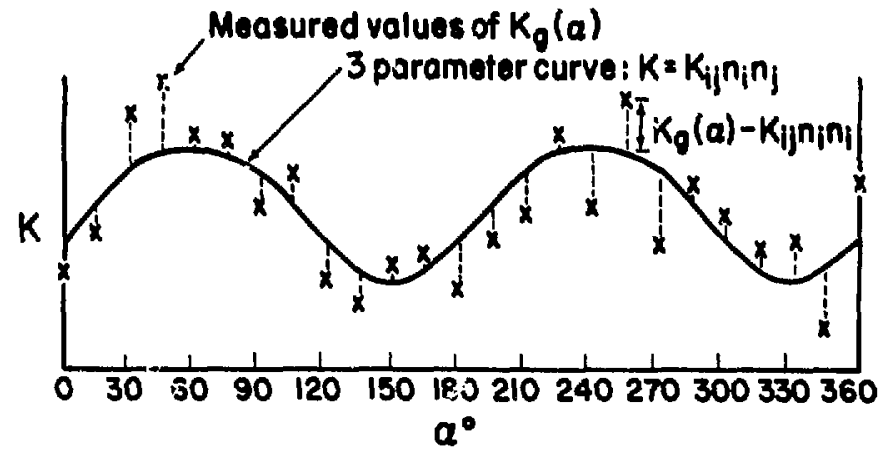

X8L817-3317

Figure 5.3. A $s ' t$ of directional permeability measurements plotted in cartesian coordinates. 
illustrates the type of three parameter curve which is fitted to the data.

A similar technique was used by Scheidegger (1954). Scheidegger minimized the function

$$
R=\sum_{m=1}^{N}\left[\left(K_{f}^{\prime}\left(\alpha_{m}\right)\right)^{-1}-\left(K_{i j}\right)^{-1} m_{i} m_{j}\right]^{2} .
$$

Although not stated by Scheidegger, this regression technique applies 10 measurements of permeability $K_{r}(\alpha)$ made in the direction of flow. Thus, $m_{i}$ is anit vector in the dineclion of now. To see this, note that permeability in the direction of flow is defined by

$$
\frac{1}{k_{f}}=\frac{J_{i} m_{i}}{q}
$$

where $q$ is the specific flux and $J_{i}$ is the gradient.

Substituting Dancy's law we have

$$
\begin{aligned}
\frac{1}{K_{i}} & =q_{i}\left(K_{i j}\right)^{-1} \frac{m_{i} q_{j}}{q}, \\
\frac{1}{K_{f}} & =\left(K_{i j}\right)^{-1} m_{j} m_{i} .
\end{aligned}
$$

So Equation $5-8$ is effectively the same as Equation 5-8, except that in $5-9, K_{i j}$ becomes the inverse of tine permeability tensor.

The solution of the regression equations is the same as the solution given by Scheidegger (1954). The equations are

$$
\begin{aligned}
& \frac{\partial R}{\partial K_{11}}=0=\sum_{n=1}^{N}-2\left[K_{2}\left(\alpha_{n}\right)-K_{i j} n_{i} n_{i}\right] \cos ^{2} \alpha_{n}, \\
& \frac{\partial R}{\partial K_{12}}=0=\sum_{n=1}^{N}-4\left[K_{2}\left(\alpha_{n}\right)-K_{i j} n_{i} n_{i}\right] \cos \alpha_{n} \sin \alpha_{n}, \\
& \frac{\partial R_{n}}{\partial K_{22}}=0=\sum_{n=1}^{N}-2\left[K_{8}\left(\alpha_{n}\right)-K_{i j} n_{i} n_{i}\right] \sin ^{2} \alpha_{n} .
\end{aligned}
$$

Rearranging, expanding $K_{i j} n_{i} n_{j}$, and putting in matrix form we have 


$$
\left[\begin{array}{lll}
\sum_{n=1}^{N} \cos ^{4} \alpha_{n} & \sum_{n=1}^{N} 2 \cos ^{3} \alpha_{n} \sin \alpha_{n} & \sum_{n=1}^{N} \sin ^{2} \alpha_{n} \cos ^{2} \alpha_{n} \\
\sum_{n=1}^{N} \cos 3 \alpha_{n} \sin \alpha_{n} & \sum_{n=1}^{N} 2 \sin ^{2} \alpha_{n} \cos ^{2} \alpha_{n} & \sum_{n=1}^{N} \sin ^{3} \alpha_{n} \cos \alpha_{n} \\
\sum_{n=1}^{N} \cos ^{2} \alpha_{n} \sin ^{2} \alpha_{n} & \sum_{n=1}^{N} 2 \sin ^{3} \alpha_{n} \cos \alpha_{n} & \sum_{n=1}^{N} \sin ^{2} \alpha_{n}
\end{array}\right]\left[\begin{array}{l}
K_{11} \\
\sum_{n=1}^{N} K_{s}\left(\alpha_{n}\right) \cos ^{2} \alpha_{n} \\
\sum_{n=1}^{N} K_{8}\left(\alpha_{n}\right) \cos _{n} \sin \alpha_{n} \\
\sum_{n=1}^{N} K_{8}\left(\alpha_{n}\right) \sin ^{2} \alpha_{n}
\end{array}\right]=
$$

Now, if for each fracture mesh, measurements are made at equal angle intervals from 0 to $2 \pi$, all sums with only odd powers of sine and cosine drop out and the equation becomes:

$$
\left[\begin{array}{ccc}
\sum_{n=1}^{N} \cos ^{4} \alpha_{n} & 0 & \sum_{n=1}^{N} \sin ^{2} \alpha_{n} \cos ^{2} \alpha_{n} \\
0 & \sum_{n=1}^{N} 2 \sin ^{2} \alpha_{n} \cos ^{2} \alpha_{n} & 0 \\
\sum_{n=1}^{N} \cos ^{2} \alpha_{n} & 0 & \sum_{n=1}^{N} \sin ^{4} \alpha_{n}
\end{array}\right]\left[\begin{array}{l}
K_{11} \\
K_{12} \\
K_{22}
\end{array}\right]=
$$

Solving for $K_{11}, K_{12}$, and $K_{22}$ gives

$$
K_{11}=\frac{\left[\sum_{n=1}^{N} K_{2}\left(\alpha_{n}\right) \sin ^{2} \alpha_{n}\right]\left[\sum_{n=1}^{N} \cos ^{2} \alpha_{n} \sin ^{2} \alpha_{n}\right]-\left[\sum_{n=1}^{N} K_{8}\left(\alpha_{n}\right) \cos ^{2} \alpha_{n}\right]\left[\sum_{n=1}^{N} \sin ^{4} \alpha_{n}\right]}{\left[\sum_{n=1}^{N} \sin ^{2} \alpha_{n} \cos ^{2} \alpha_{n}\right]^{2}+\left[\sum_{n=1}^{N} \cos ^{4} \alpha_{n}\right]\left[\sum_{n=1}^{N} \sin ^{4} \alpha_{n}\right]}
$$




$$
\begin{aligned}
& K_{12}=\frac{\sum_{n=1}^{N} K_{8}\left(\alpha_{n}\right) \cos \alpha_{n} \sin \alpha_{n}}{\sum_{n=1}^{N} 2 \sin ^{2} \alpha_{n} \cos ^{2} \alpha_{n}} . \\
& K_{22}=\frac{\sum_{n=1}^{N} K_{8}\left(\alpha_{n}\right) \cos ^{2} \alpha_{n}}{\sum_{n=1}^{N} \sin ^{2} \alpha_{n} \cos ^{2} \alpha_{n}} \\
& -\frac{\left[\sum_{n=1}^{N} K_{n}\left(\alpha_{n}\right) \sin ^{2} \alpha_{n}\right]\left[\sum_{n=1}^{N} \cos ^{2} \alpha_{n} \sin ^{2} \alpha_{n}\right]-\left[\sum_{n=1}^{N} K_{8}\left(\alpha_{n}\right) \cos ^{2} \alpha_{n}\right]\left[\sum_{n=1}^{N} \sin ^{4} \alpha_{n}\right]}{\left[\sum_{n=1}^{N} \sin ^{2} \alpha_{n} \cos ^{2} \alpha_{n}\right]^{2}+\left[\sum_{n=1}^{N} \cos ^{4} \alpha_{n}\right]\left[\sum_{n=1}^{N} \sin ^{4} \alpha_{n}\right]} \\
& \text { - } \frac{\sum_{n=1}^{N} \cos ^{4} \alpha_{n}}{\sum_{n=1}^{N} \sin ^{2} \alpha_{n} \cos ^{2} \alpha_{n}} \text {. }
\end{aligned}
$$

ELLFMG first reads the directional permeabilities for the flow region, and for the study region(s) if any had been specified by the user. The sums of the various trigonometric terms in Equation 5-18, 5-19 and 5-20 are computed by subroutine SUM. Then $K_{11}, K_{12}$, and $K_{22}$ are computed using these equations.

\subsection{Principal Permeabilities and Directions}

Knowing the values of $K_{11}, K_{12}$ and $K_{22}$ the values and directions of the principal permeabilities $K_{1}$ and $K_{2}$ can be calculated with standard techniques of linear algebra. The techniques are given here only for completeness. In Edelen and Kydonicfs (1972) we have

$$
\mathbf{K}_{\mathrm{ij}} \mathbf{E}_{\mathbf{j}}=\lambda \mathrm{E}_{\mathrm{i}} \text {, }
$$

where $E_{i}$ is a unit vector in a principal direction, or eigenvector, for $K_{i j}$. The transformation $\mathrm{K}_{\mathrm{j} j} \mathrm{E}_{\mathrm{j}}$ gives a vector in the same direction as $\mathrm{E}_{\mathrm{j}}$, but of magnitude $\lambda$ where $\delta_{\mathrm{j} j}$ is the Kronecker delta. Thus,

$$
\left(K_{i j}-\lambda \delta_{i j}\right) E_{j}=0
$$

Here the components of $E_{j}$ and $\lambda$ are unknowns. This equation can have a solution only if

$$
\left|\begin{array}{ll}
K_{11}-\lambda & K_{12} \\
K_{12} & K_{22}-\lambda
\end{array}\right|=0
$$


or

$$
\lambda^{2}-\left(\mathbf{K}_{11}+\mathbf{K}_{22}\right) \lambda+\mathbf{K}_{11} \mathbf{K}_{22}-\mathbf{K}_{12}^{2}=0 .
$$

So the principal permeabilities are

$$
\begin{aligned}
& K_{1}=\lambda_{1}=\frac{K_{11}+K_{22}}{2}+\frac{\sqrt{\left(K_{11}+K_{22}\right)^{2}-4\left(K_{11} K_{22}-K_{12}^{2}\right)}}{2} \\
& K_{2}=\lambda_{2}=\frac{K_{11}+K_{22}}{2}-\frac{\sqrt{\left(K_{11}+K_{22}\right)^{2}-4\left(K_{11} K_{22}-K_{12}^{2}\right)}}{2}
\end{aligned}
$$

The principal directions, $E 1_{j}$ and $E 2_{j}$, are found by solving the equations

$$
\left(K_{i j}-\lambda_{1} \delta_{i j}\right) E 1_{j}=0
$$

and

$$
\left(K_{i j}-\lambda_{2} \delta_{i j}\right) E 2_{j}=0 \text {, }
$$

for the components of the $K 1_{j}$ and $E 2_{j}$.

Let $E_{j}=\left[\begin{array}{l}x \\ y\end{array}\right]$. Now for each $\lambda$ we have

$$
\left[\begin{array}{ll}
K_{11}-\lambda_{i} & K_{12} \\
K_{12} & K_{22}-\lambda_{i}
\end{array}\right]\left[\begin{array}{l}
x \\
y
\end{array}\right]=0
$$

Using row reduction we obtain

$$
\left[\begin{array}{ll}
1 & \frac{K_{12}}{K_{11}-\lambda_{i}} \\
0 & \left(K_{22}-\lambda_{i}\right)-\frac{K_{12}^{2}}{K_{11}-\lambda_{i}}
\end{array}\right]=\left[\begin{array}{cc}
1 & \frac{K_{12}}{K_{11}-\lambda_{i}} \\
0 & 0
\end{array}\right]
$$

because $\left(K_{11}-\lambda_{i}\right)\left(K_{22}-\lambda_{i}\right)-K_{12}^{2}=0$ due 10 the choice of $\lambda$ (Equation 5-24). So we can choose

$$
\begin{aligned}
& x=1, \\
& y=\frac{\lambda_{i}-K_{11}}{K_{12}}=\frac{K_{12}}{\lambda_{i}-K_{22}} .
\end{aligned}
$$

The $\mathrm{E}_{\mathrm{i}}$ can be expressed as the unit vectors

$$
\begin{aligned}
& E 1_{i}=\frac{1}{\sqrt{1+\left(\frac{\lambda_{1}-\lambda_{11}}{K_{12}}\right)^{2}}}, \frac{\left(\lambda_{1}-K_{11}\right) / K_{12}}{\sqrt{1+\left(\frac{\lambda_{1}-K_{11}}{K_{12}}\right)^{2}}}, \\
& E 2_{i}=\frac{1}{\sqrt{1+\left(\frac{\lambda_{2}-\lambda_{11}}{K_{12}}\right)^{2}}}, \frac{\left(\lambda_{2}-K_{11}\right) / K_{12}}{\sqrt{1+\left(\frac{\lambda_{2}-K_{11}}{K_{12}}\right)^{2}}},
\end{aligned}
$$


If $\lambda_{1}=\lambda_{2}$, the ellipse is circular and any two perpendicular vectors can be eigenvectors. In this case we can choose

$$
\begin{aligned}
& E 1_{j}=(0,1) \\
& E 2_{j}=(1,0) .
\end{aligned}
$$

The eigenvalues and eigendirections are compuled using these results.

\subsection{Mean Square Error}

The mean square error, MSE, is simply given by

$$
\begin{aligned}
& \text { MSE }=\frac{R}{N} \\
& \text { MSE }=\frac{1}{N} \sum_{n=1}^{N}\left[K_{2}\left(\alpha_{n}\right)-\left[K_{11} \cos ^{2} \alpha_{n}+2 K_{12} \cos \alpha_{n} \sin \alpha_{n}+K_{22} \sin ^{2} \alpha_{n}\right)\right]^{2}
\end{aligned}
$$

In order to use the MSE to compare the data from different fracture samples the MSE must be normalized as follows.

$$
\begin{aligned}
& \text { NMSE }=\frac{M S E}{K_{1} K_{2}} \\
& \text { NMSE }=\frac{1}{N K_{1} K_{2}} \sum_{n=1}^{N}\left[K_{8}(\alpha)-\left[K_{11} \cos ^{2} \alpha_{n}+2 K_{12} \cos \alpha_{n} \sin \alpha_{n}+K_{22} \sin ^{2} \alpha_{n}\right]\right]^{2} .
\end{aligned}
$$

As NMSE approaches zero, the fracture systems behave more like anisotropic, homogeneous porous media. But this normalization creates a problem for very anisotropic results. If the lower principal permeability tends to zero, NMSE as defined above tends to infinity, resulting in an overflow of the compuler. In this case, the definition of NMSE is slighty modified. Instead of using the product of the principal permeabilities, i.e. the square of their geometric mean, the program uses the square of the arithmetic mean:

$$
\text { NMSE }=\frac{\text { MSE }}{\left[\left(K_{1}+K_{2}\right) / 2\right]^{2}}
$$

ELLFMG then outputs the best-fit tensor characteristics and the mean square error. One or more files are also printed to serve as an input for plotting both the best-fit ellipse and the directional permeability results. One file is printed for the flow region, and one file is printed for each gradient type (local or global) specified for each subregion, if relevant. 


\subsection{PLOTTING PROGRAMS ELLP AND DIMES}

ELLP and DIMES help the user visualize the outputs of the chain of programs. ELLP plots the end result after processing by FMG, RENUM, LINEL and ELLFMG is completed. The equivalent permeability tensor is represented in both polar and cartesian coordinates. DIMES plots the line network at various stages of the process.

\subsection{ELLP}

The input to ELLP (ELLipse Plot) is constituted by one or more files written by program ELLFMG. The user does not have to write any input file. The file ELLIPSE.PLT contains input relative to the flow region computations. If study regions were specified by the users, ELLIPSEG01.PLT, ELLIPSEL01.PLT, etc. ... contain input relative to the study region computations, using the global and local gradient respectively (see Section 4.3.2). Each of these files

contains, in polar coordinates, the pairs (alpha, $1 / \sqrt{\mathrm{K}_{2}}$ ) defining the best-fit cllipse and the directional permeabilities compuled by LINEL. Also input are the direction of the principal permeabilities and the values of these principal permeabilities.

ELLP determines what the maximum value of $1 / \sqrt{\mathrm{Kg}}$ is, computes a corresponding scale for the plots. The computed and fitted values are then plotted, both in polar coordinates and in cartesian coordinates. Figure 6.1 gives an example of such a plot.

\subsection{DIMES}

DIMES plots the fracture networks. As can be seen in Figure 1-1 in the Introduction, DIMES (DIsc MEShes) can accept plot files from FMG, RENUM, FMG3D, or CHANGE. When the program is called, it looks for input files in the current computer directory. If files named DIMES01.DAT, DIMES02.DAT, etc. ..., are present, then the network to be plotted is three-dimensional. Otherwise it is two-dimensional. The three-dimensional mode of operation 

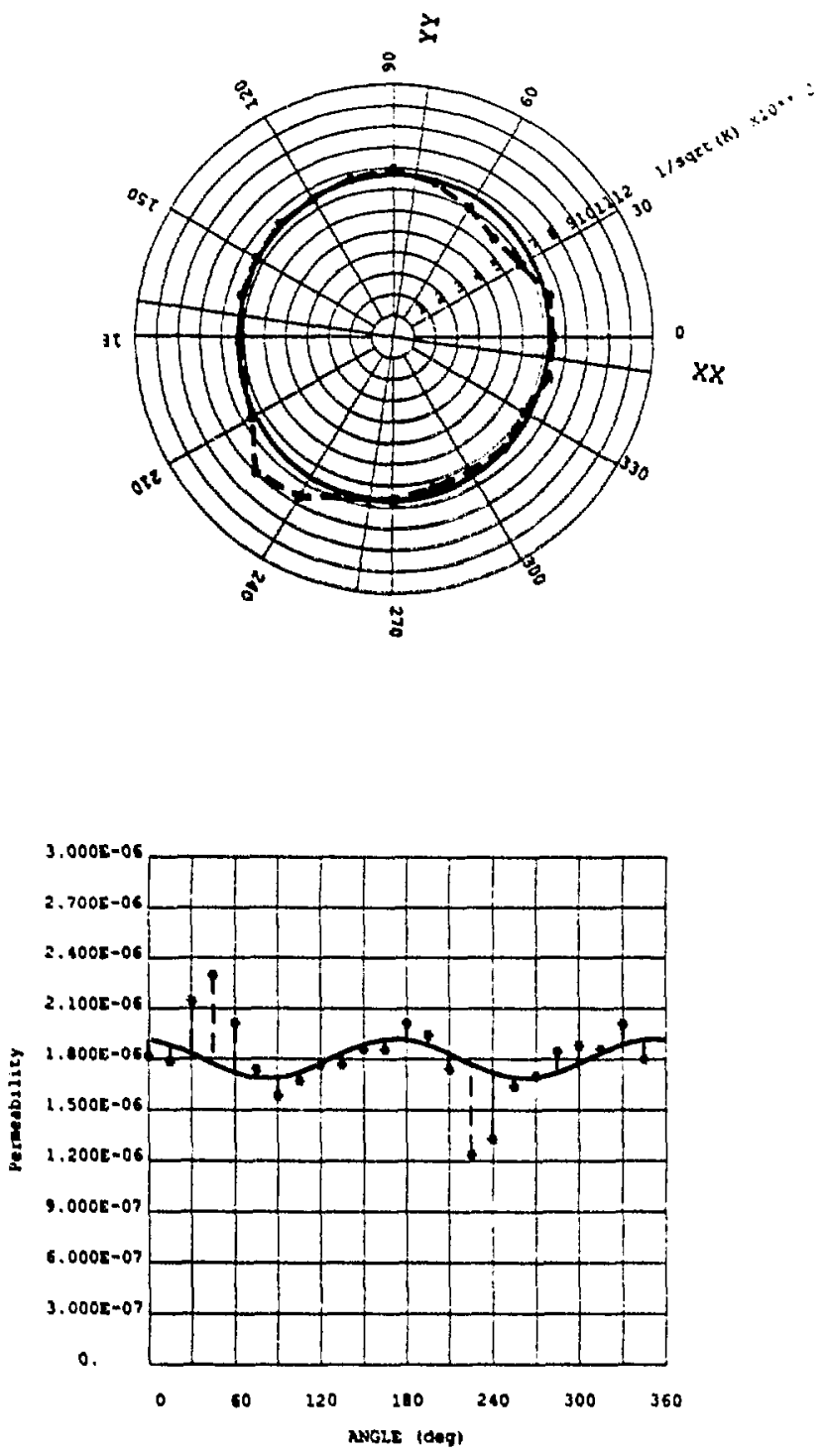

PERMEABILITY ELLIPSE

( nmse $=0.1313 E-01$ )

Figure 6.1. Polar and cartesian plots of directional permeabilities. 
of DIMES is documented in Gilmour et al. (1986a and b). It draws a disk network and the intersections between the discs, from any point of view specified by the user.

A simple option has been added to DIMES in order to also handle two-dimensional networks. If files named LINESO1.DAT, LINES02.DAT, etc. ..., are present, then these specify a network of line elements generated either by FMG or by the three-dimensional channel generator CHANGE (Billaux, ot al., 1988). In the two-dimensional case, DIMES reads the tille of the plot, the size of the generation region, the angle and size of the flow region, from the files RENUMGR.DAT, RENUM00.DAT, RENUM01.DAT, etc. ... created by FMG. The coordinates of the endpoints of the line segment are then read and all the segments are plotted. The relative disposition of the generation region and the flow region is plotted in a small box in the lower left comer. If only one region is plotted, it means the region being plotted is the generation region itself. Figures 6.2 and 6.3 show the network corresponding to the the permeability plot in Figure 6.1. 

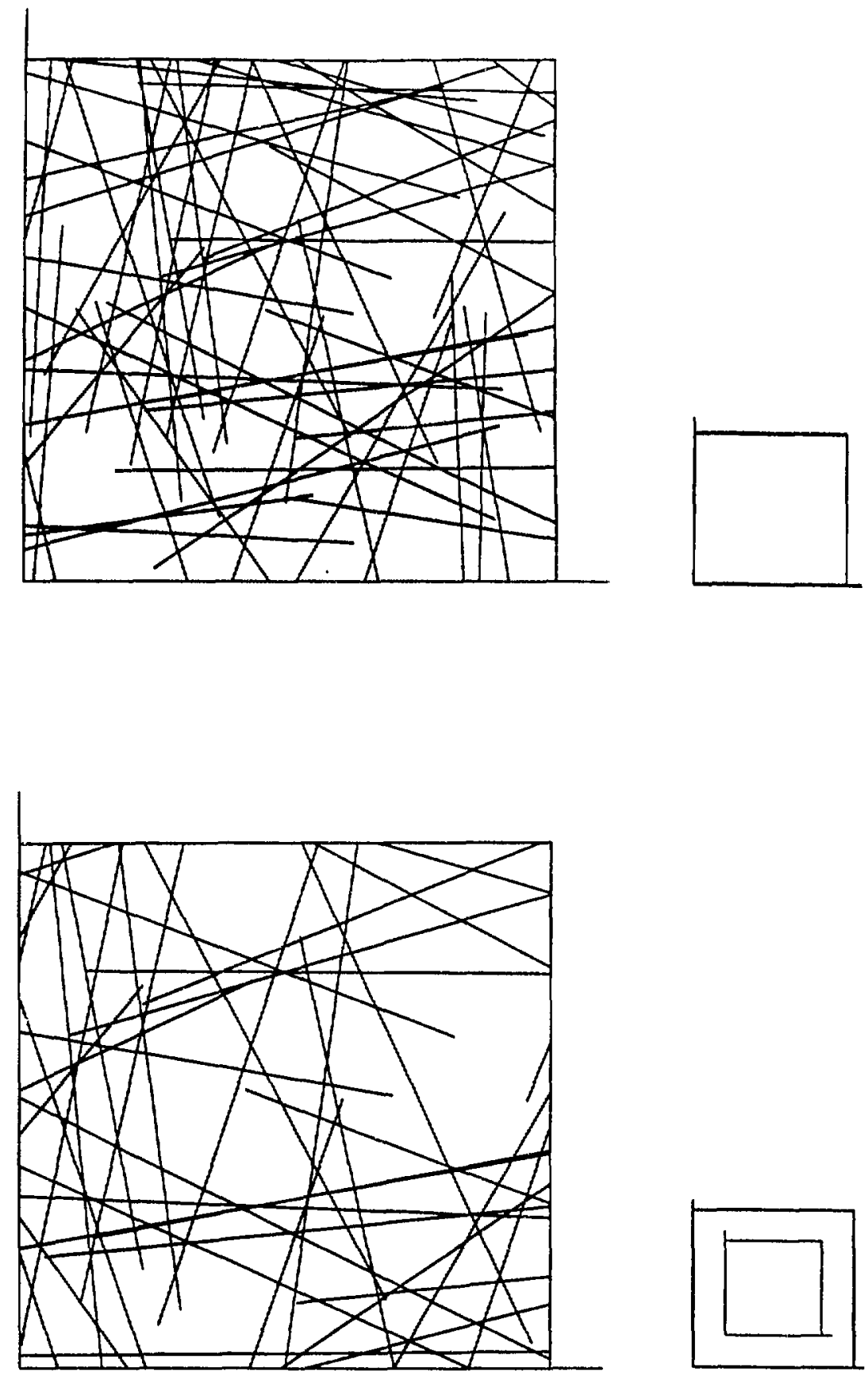

Figure 6.2. Fractures in the generation region, and the $0^{\circ}$ rotation flow region. 

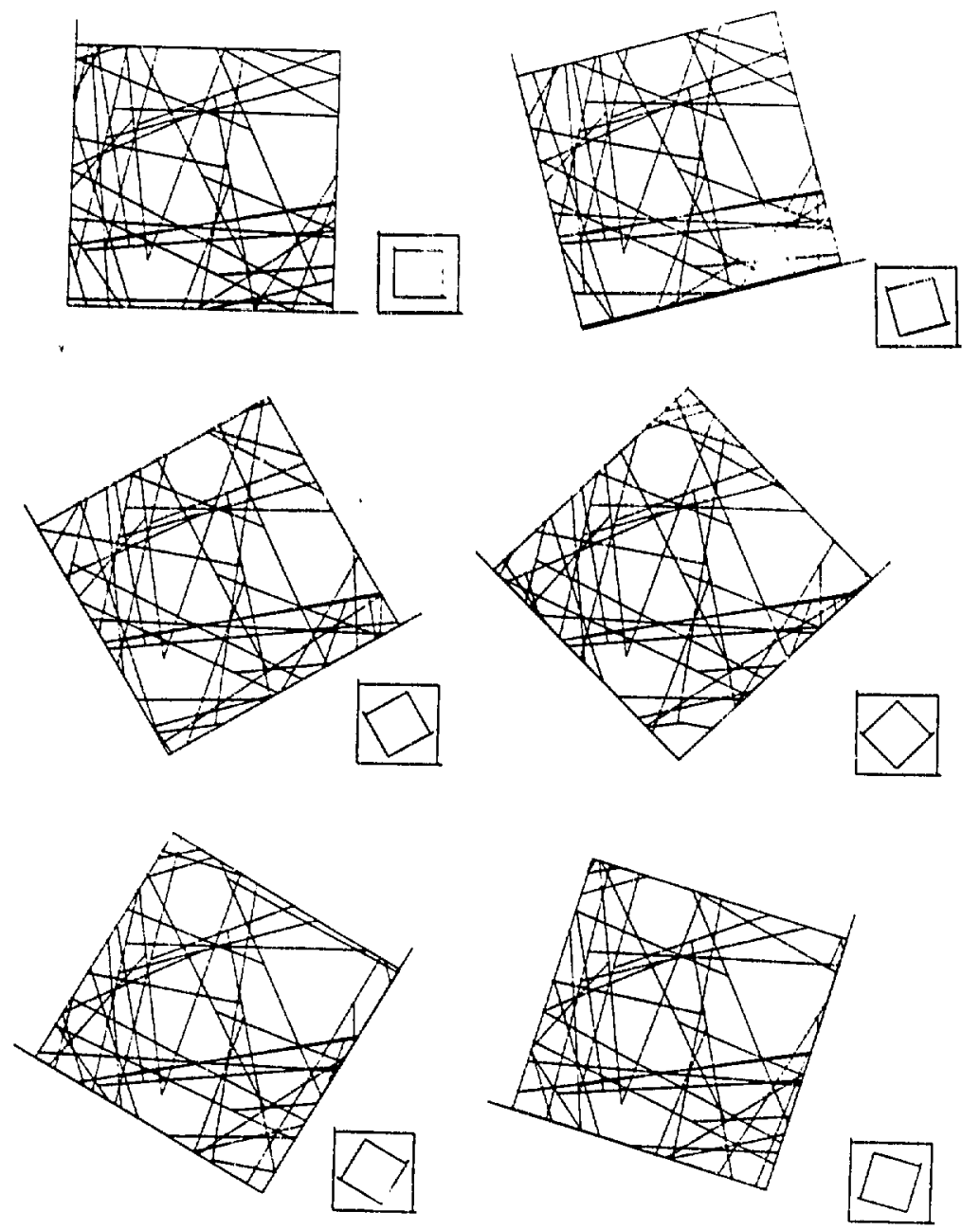

KBL ดaj-897

Figure 6.3. Line network in flow regions. 


\subsection{REFERENCES}

Billaux, D. and P. Fuller (1988). An algorithm for mesh simplification applied to fracture hydrology, Journal of the International Association for Mathematical Geology, New York (accepted for publication).

Billaux, D. and J. C. S. Long (1988a). CHANGE: A numerical model for three-dimensional modelling of channelized flow in rock. Theory and design, Lawrence Berkeley Laboralory Report LBL-24910.

Billaux, D. and J. C. S. Long (1988b). CHANGE: A numerical model for three-dimensional modelling of channelized Mow in rock. User's manual and listing, Lawrence Berkelcy Laboratory Report LBL-24911.

Edelin, D. and A. Kydoniefs (1972). An Introduction to Linear Algebra, American Elsevier, Inc., New York, New York.

Gilmour, P., D. Billaux and J. C. S. Long (1986a). Models for calculating fluid flow in randomly generated three-dimensional networks of disc-shaped fractures. Theory and design of FMG3D, DISCEL, and DIMES, Lawrence Berkeley Laboratory Report Number 19515, $143 \mathrm{pp}$.

Gilmour, P., D. Billaux and J. C. S. Long (1986b). Models for calculating fluid flow in randomly generated three-dimensional networks of disc-shaped fractures. User Manuals and listings for FMG3D, DISCEL, and DIMES, Lawrence Berkeley Laboratory Report Number 19516.

Hammersly, J. M. and D. C. Handscomb (1964). Monte Cario Methods, Methuen and Co., London, 178 pp.

Karasaki, K. (1987). A new advection-dispersion code for calculating transport in fracture networks, Lawrence Berkeley Laboratory Earth Science Division 1986 Annual Report, LBL Report Number 22090, Berkeley, pp 55-57.

Long, J. C. S. (1983). Investigation of Equivalent Porous Medium Permeability in Networks of Discontinuous Fractures, Ph.D. Thesis, College of Engineering, University of California, Berkeley, 277 pp.

Long, J. C. S., J. S. Remer, C. R. Wilson, P. A. Witherspoon (1982). Porous media equivalents for networks of discontinuous fractures, Water Resources Research, 18 (3), pp. 645-658. 
Long, J. C. S., P. A. Witherspoon (1985). The relationship of the degree of interconnection and permeability in a fracture network, Journal of Geophysical Research, 90 (B4), Pp. 3087-3098.

Robinson, P. C. (1982). NAMNET - Network flow program, AERE Harwell Report Number 209-81-7-WAS UK, 28 pp.

Scheidegrer, A. E. (1954). Directional permeability of porous media to homogeneous fluids, Geofisica pura Applicata, 28, pp. 75-90.

Wilson, C. R. (1970), An Investigation of Laminar Flow in Fractured Porous Rocks, Ph.D. Thesis, University of Califomia, Berkeley, 178 pp. 